“ (C) 2017 IEEE. Personal use of this material is permitted. Permission from IEEE must be obtained for all other uses, in any current or future media, including

reprinting/republishing this material for advertising or promotional purposes, creating new collective works, for resale or redistribution to servers or lists, or reuse of any copyrighted component of this work in other works." 


\title{
Design and Implementation of an Amorphous High-Frequency Transformer Coupling Multiple Converters in a Smart Microgrid
}

\author{
Mohammad Jafari, Member, IEEE, Zahra Malekjamshidi, Student Member, IEEE, \\ Gang Lei, Member, IEEE, Tianshi Wang, Glenn Platt, and Jianguo Zhu, Senior Member, IEEE
}

\begin{abstract}
Recent improvements in magnetic material characteristics and switching devices have generated a possibility to replace the electrical buses with highfrequency magnetic links in microgrids. Multiwinding transformers (MWTs) as magnetic links can effectively reduce the number of conversion stages of renewable energy system by adjusting turn ratio of windings according to the source voltage level. Other advantages are galvanic isolation, bidirectional power flow capability, and simultaneous power transfer between multiple ports. Despite the benefits, design, and characterization of MWTs are relatively complex due to their structural complexity and cross-coupling effects. This paper presents all stages of numerical design, prototyping, and characterization of an MWT for microgrid application. To design the transformer for certain value of parameters, the reluctance network method is employed. Due to the iterative nature of transformer design, it presented less computation time and reasonable accuracy. A prototype of designed transformer is implemented using amorphous magnetic materials. A set of experimental tests are conducted to measure the magnetic characteristics of the core and series coupling and open-circuit tests are applied to measure the transformer parameters. A comparison between the simulation and experimental test results under different loads within the medium-frequency range validated both design and modeling procedures.
\end{abstract}

Index Terms-Design, modeling, multiwinding, reluctance network method (RNM), smart microgrid, transformer.

\section{INTRODUCTION}

$\mathbf{R}$ ECENT advancements on smart microgrid technologies have imposed increasing demands for more reliable and flexible converters and control techniques. In contrast to the traditional electrical ac and dc buses, the high-frequency magnetic links can reduce effectively the number of conversion stages in microgrids with the help of modern soft magnetic materials with

Manuscript received January 29, 2016; revised April 4, 2016; accepted April 28, 2016.

M. Jafari, Z. Malekjamshidi, G. Lei, T. Wang, and J. G. Zhu are with the School of Electrical, Mechanical and Mechatronic Systems, University of Technology Sydney, Sydney, NSW 2007, Australia (e-mail: Mohammad.Jafari@uts.edu.au; Z.Malekjamshidi@student.uts.edu.au; Gang.Lei@uts.edu.au; Tianshi.Wang@student.uts.edu.au; Jianguo. Zhu@uts.edu.au).

G. Platt is with the Commonwealth Scientific and Industrial Research Organisation (CSIRO), Newcastle, NSW 2304, Australia (e-mail: Glenn.Platt@csiro.au). superior magnetic characteristics and fast- and low-power loss switching devices [1]-[5]. Multiwinding transformers (MWTs) can provide a common magnetic bus for integrating renewable energies in the form of magnetic flux. Their application in the multiactive bridge phase-shift converter makes it possible to simply integrate the sources of different voltage levels using different turn ratios [6]-[9]. Other advantages are galvanic isolation, bidirectional power flow capability, faster control, and simultaneous power transfer among the ports [10]-[12]. Design of MWTs for certain value of inductances is relatively complex due to their complex structureand cross-coupling effects [13]-[15]. Research on MWTs mainly is focused on their characterization and modeling and there is not much research on design methods [16]-[19]. This paper provides a complete discussion on design, prototyping, and experimental tests of a high-frequency toroidal MWT. The transformer is designed for certain values of inductances, using reluctance network method (RNM). A prototype has been fabricated using amorphous magnetic materials to validate the accuracy of proposed design method. To measure the transformer parameters and extract the equivalent electrical model, the open circuit, differential and cumulatively coupled tests are conducted on the prototype transformer. The short-circuit test is excluded as it did not provide reliable results due to relatively high-leakage inductances. The prototype transformer is tested for a wide-frequency range under different load conditions and the results are compared with the simulation based on the extracted transformer model.

\section{Multiwinding Transformer as COMmon Magnetic LINK}

MWTs have been used as the common magnetic links in multiactive bridge phase-shift converters to integrate the renewable energies effectively [20]-[23]. The converter designed in this research includes four ports connected to the load, fuel cell, battery, and photovoltaic (PV) as illustrated in Fig. 1. The Hbridge units produce high-frequency ac square wave from dc buses linked to the dc sources. The power flow between the ports one, two, and three is controlled by using the phase-shift technique. To apply the technique, port one is selected as the reference and ports two and three are shifted for a leading or lagging phase angle to send or receive power to port one. A dutycycle control is applied to port three for the maximum power point tracking of PV panel. As illustrated in the figure, port one is a bidirectional port transferring power from renewable 


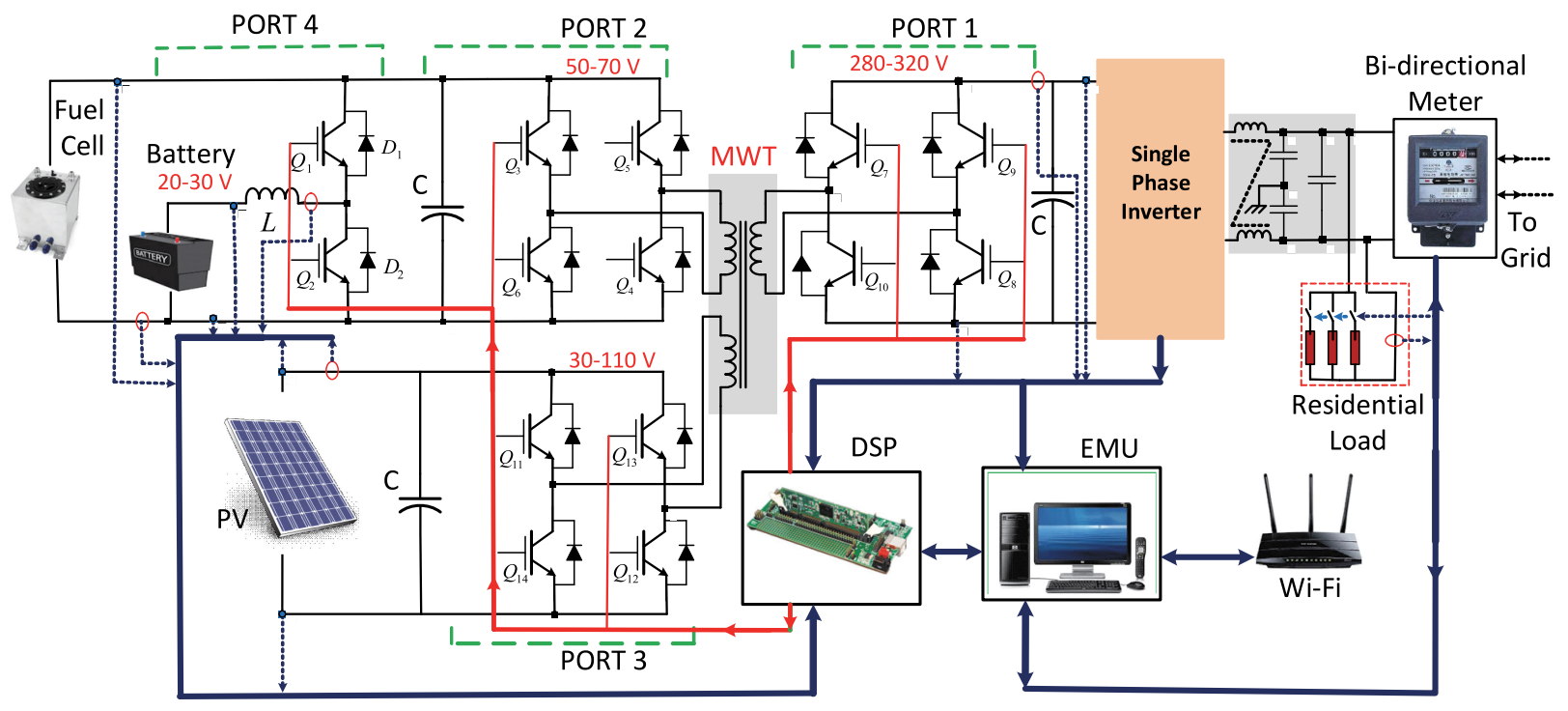

Fig. 1. Structure of proposed multiactive bridge phase-shift converter.

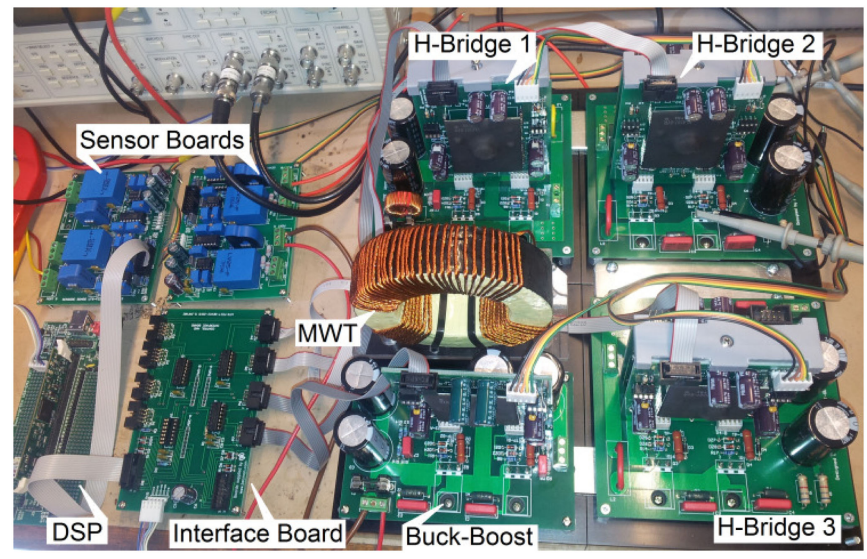

Fig. 2. Experimental set of designed renewable energy system.

sources or battery to the inverter and further to the load and grid. It can also transfer the power reversely from the grid to the battery (using port two and four). Port two is connected to the dc bus linking directly to the fuel cell and a bidirectional buck-boost converter linked to the $24-\mathrm{V}$ battery bank. The voltage of the dc bus is $50-70 \mathrm{~V}$ as it is connected directly to the fuel cell stack. It operates in the buck and boost modes to charge and discharge the battery, respectively. Fuel cell in this system can be used as back up energy source and in normal operation mode, power transfers from PV (port three) as preferred source to the inverter and load (port one). Battery can be charged by fuel cell, PV, or grid and also is used to balance the voltage of dc bus due to low dynamic response of fuel cell during fuel cell operation. The experimental set of designed renewable energy system including MWT is presented in Fig. 2. The MWT as a common magnetic link provides flexibility in the direction of power flow between the ports. It facilitates the operation of system in different modes based on direction of power flow and applying various energy management scenarios in both gridconnected and islanding modes. The leakage inductances of the transformer windings are used as energy transfer components in this topology [7]-[12]. Using external bulked inductors in series with transformer windings is suggested although it increases the system size and cost [9], [10]. To avoid this problem, the MWT should be designed for certain value of inductances according to the desired range of operating phase shift at the required power throughput. Detail of the converter operation modes is out of scope of this paper as this paper mainly focuses on the design, modeling, and experimental test of the high-frequency MWT.

\section{DESIGn OF MULTIWINDING TRANSFORMER}

Design of MWTs for certain value of inductances using the classical methods of transformer design is not accurate due to their structural complexity and cross-coupling effects. Numerical methods such as 2-D and 3-D finite-element methods (FEMs) or RNM are commonly used in design of magnetic structures such as electrical motors and transformers. The FEM is an accurate field analysis method but when used in design optimization, the computational cost is too high. On the other hand, the RNM is very fast but less accurate [24]-[29]. Magnetic field analysis using an FEM can take into account the nonlinearity of magnetic materials, geometry, and actual winding distribution while an RNM is based on linear assumptions. The RNM also can be used for analysis of devices with arbitrary geometries and excitations using a general 3-D element and variable size reluctance network [30]. In this research, the RNM is used in the design process. Due to iterative nature of the transformer design, it presented less computation time and reasonable accuracy. To use it in design optimization, various methods of choosing proper calculation domains such as number of 3-D elements and dimension of field analysis area have been implemented to improve its accuracy. The required values of leakage and self-inductances of transformer windings are selected as design specifications. The number of winding turns, dimension of magnetic core and thickness of insulator between each winding and the core are selected as design variables. To reduce the total time of design, initial values of design variables are defined using classical methods of the transformer design [31]. To find the initial size of the core, the area-product was defined based on the power handling 


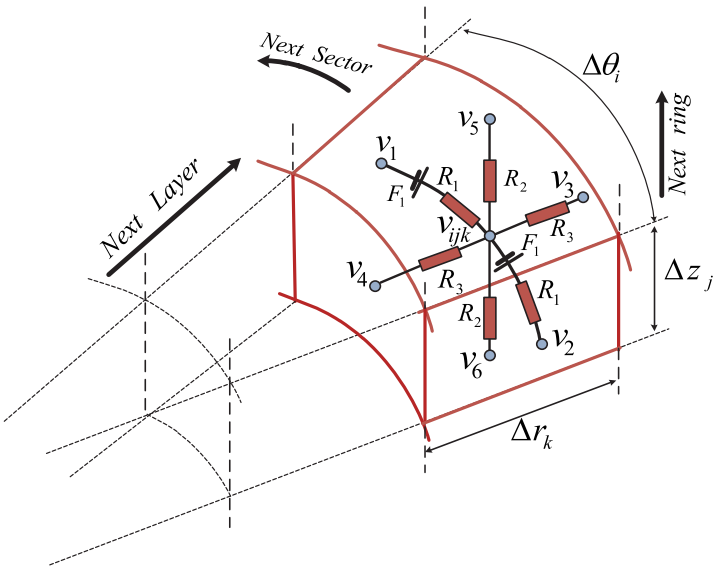

Fig. 3. 3-D reluctance element.

capacity, current density, and flux density. The resultant value then was used to find the core size from area-product/core-size charts. Finally the number of turns was calculated using Faraday's law. To analyze the magnetic field using an RNM, the magnetic structure is divided into three different areas including magnetic core, insulator, and free space around the core. To adjust the leakage inductance of transformer, the thickness of the insulator between windings and the magnetic core considering isolation constraints are selected as variables. To do the analysis, the magnetic structure is assumed to operate in linear section of the magnetization curve, and therefore, a constant value of permeability is used. The magnitude of terminal voltage is chosen such that the peak flux density would just reach the knee point of the magnetization curve, and thus, the linear model can give sufficient accuracy. This is particularly true for the magnetic material used in this design (amorphous 2605SAl) based on data provided by factory and experimental tests presented in Section IV. This can effectively avoid the extra current harmonics and excessive magnetization current and core losses due to the distortion of magnetic field caused by the nonlinear magnetization curve. On the other hand, because of the full use of the linear region, there is no need to use bigger core or more winding than necessary. This assumption also helps to represent the magnetic behavior of the core under nonsinusoidal excitation currents applying the superposition rule to Fourier's terms of the current. Due to the axial symmetry of the toroidal core, the cylindrical coordinate system has been selected for numerical modeling. The core is partitioned into thousands of 3-D elements. Each element includes six reluctances in basic directions of cylindrical coordination, $r, \theta$, and $Z$, and is presented by $Q_{i j k}$. The indexes $i, j$, and $k$ represent the unique situation of 3-D element among the entire magnetic structure. All six branches are connected to a central node at one side and to the boundary nodes at boundary surfaces of 3-D element at the other side as shown in Fig. 3. The reluctances are calculated using geometrical shape and permeability factor of the element and sources, $F_{i j k}$, are representative of magnetic motive force, $m m f$, in the corresponding direction.

The sources should be equal to zero in directions with no magnetic flux, which makes the element simpler for analysis.
The magnetic structure will be divided into thousands of such elements arranged in sectors in $\theta$, layers in radial $r$, and rings in $Z$-directions. The value of voltage sources, $F_{i j k}$ in case of toroidal core in directions of $r$ and $Z$ is almost zero and in $\theta$ direction depends on the winding turns covering the element and the current flowing through it. Applying the nodal analysis to the central node of elements results a group of equations for the central node voltages as unknowns. The final equation of entire magnetic structure considering the equations of all nodes can be written in matrix form as

$$
Y^{\prime} \tilde{V}-Y \tilde{F}=0
$$

where $Y^{\prime}, Y, \tilde{V}$, and $\tilde{F}$ are central-node-linked conductance, source-linked conductance, central-node voltage, and source voltage matrices, respectively. To solve the aforementioned equation and define the potential of all central nodes of the 3-D elements an initial condition is considered. The potential of the central nodes of the elements situated on the last layer and rings are assumed to be zero. The number of nodes and the number of 3-D elements depend on the number of selected divisions in three dimensions of cylindrical coordination. The value of current between the nodes representing the magnetic flux can be calculated using the potential of central nodes of adjacent elements and their interconnected resistances. Considering all branches of the element in three directions, one can calculate the field intensity $H_{i}$, the branch volume $V_{i}$, and the flux density $B_{i}$ for $i=1,2, \ldots, 6$ as [30]

$$
\begin{aligned}
H_{i} & =\left(v_{i j k}-v_{i}\right) / l_{i} \\
V_{i} & =A_{i} l_{i} \\
B_{i} & =\varphi_{i} / A_{i} .
\end{aligned}
$$

The closed-form volumetric integration of the distributed stored energy can be calculated from

$$
W_{S}=\int_{V} \frac{H \cdot B}{2} d V=\frac{\mu}{2} \int_{V}|H|^{2} d V
$$

where $W_{S}$ is the stored energy in a 3-D element enclosed by volume $V$, and $\mu$ the magnetic permeability [30]. The net magnetic energy stored in the $i$ th branch of the element $Q_{i j k}$ based on (2)-(5) assuming constant flux density in the element can be calculated using the following expression:

$$
W_{i}=\left(\mu V_{i} H_{i}^{2}\right) / 2
$$

substituting (2)-(4) into (6) results in

$$
W_{i}=\left(v_{i j k}-v_{1 i j k}-F_{i}\right)^{2} / 2 R_{i}
$$

where $R_{i}$ is the reluctance of the respected branch and the value $F_{i}$ should be equal to zero for branches without $m m f$. The overall energy of a 3-D element $Q_{i j k}$, can be obtained by integrating of the energy in all six branches. To calculate the winding self-inductances, a closed surface enclosing entire magnetic structure is assumed. The total enclosed energy is calculated by integrating the energy of all $n$ elements inside the surface. The resultant total energy, $W_{S}$, can be used to define the equivalent inductance, $L_{\text {eq }}$, using

$$
L_{\mathrm{eq}}=2 W_{s} / I_{s}^{2} .
$$




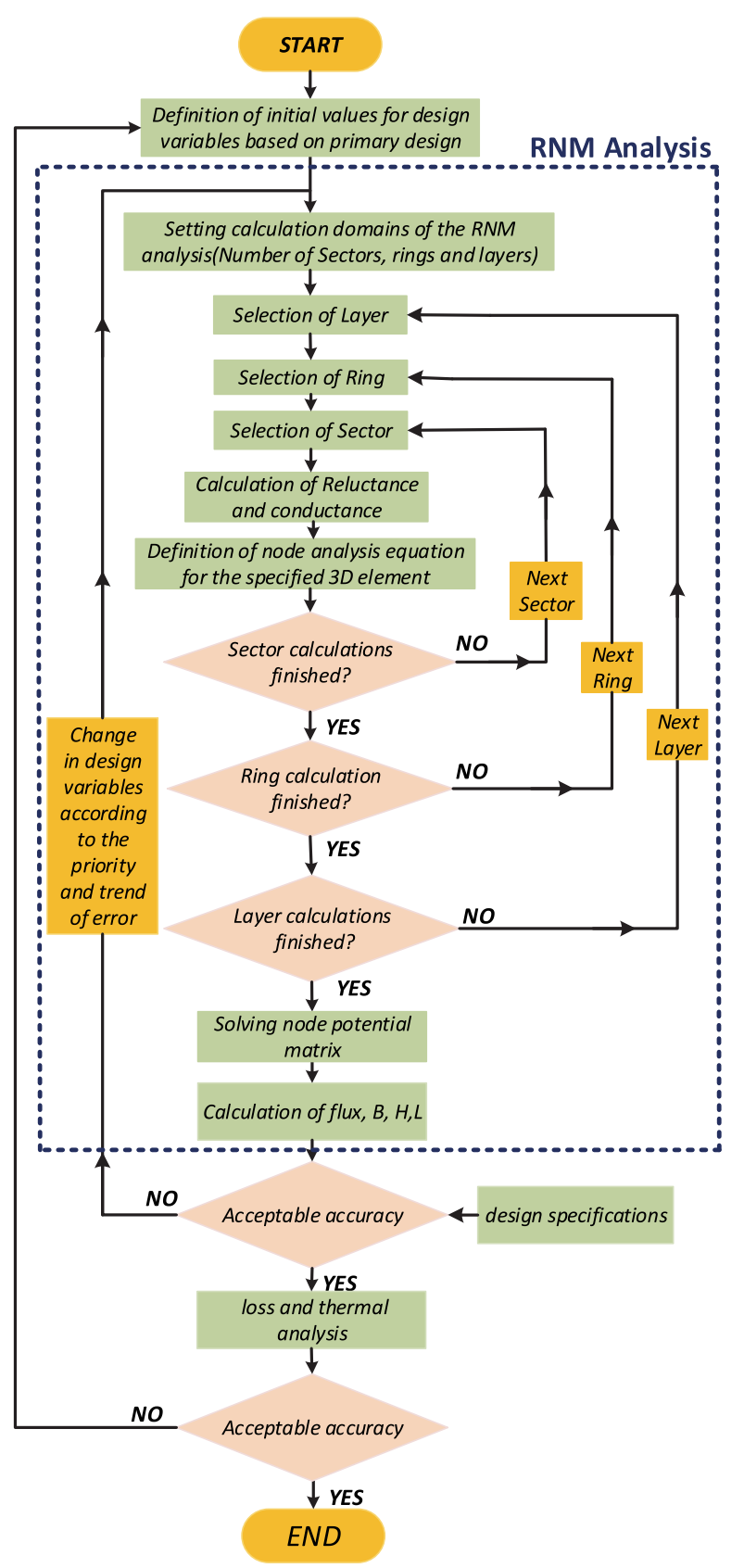

Fig. 4. Flow chart of transformer design process using MATLAB.

The self-inductance of windings one $L_{11}$, two $L_{22}$, and three $L_{33}$ was calculated based on (8). To define the leakage inductances of the windings, $L_{l 1}, L_{l 2}$, and $L_{l 3}$, two different enclosed surfaces, one includes only the core area and the other enclosed core, insulator and surrounding areas (including leakage flux distribution area) are considered and the difference between the energies stored within the volumes enclosed by these surfaces can be used. The values of self- and the leakage inductances of all windings of the MWT are calculated by using the method outlined previously. The core loss resistance for the resultant structure can be found using the Steinmetz equation based on the flux density and frequency. Fig. 4 presents the flow chart showing stages of analysis using MATLAB. As can be seen in

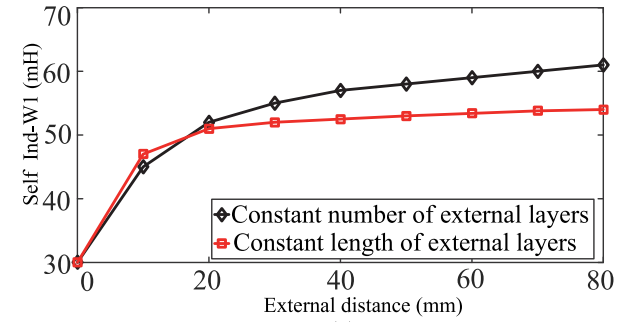

(a)

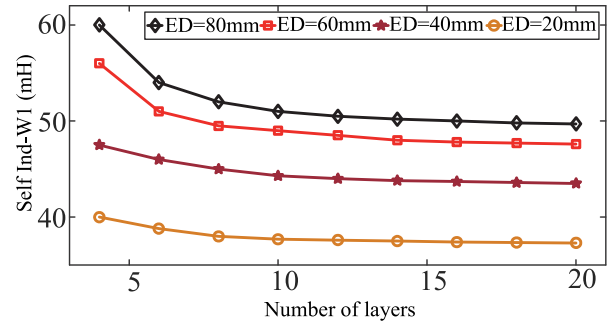

(b)

Fig. 5. (a) Self-inductance of winding one for two cases of fixed and stretchable layer selection. (b) Self-inductance of winding one for various external distances.

the figure, the parts enclosed by dashed line are steps of RNM analysis. The calculation domains such as number of sectors, rings, and layers are defined at first step. The analysis starts with the first sector of the first ring of the first layer and the next ring is selected when all sectors are analyzed and the next layer is selected when analysis of all rings of the previous layer is completed. The value of $B, H$, and $L$ are calculated based on (2)(8). The resultant values are compared with specifications, and in case of excessive error, the design variables are changed according to the priority. In case of acceptable results, the magnetic structure is analyzed for losses and thermal considerations. The loss analysis was carried out based on the Steinmetz equation. To calculate the copper losses, both skin and proximity effects are considered. To improve the accuracy analysis, various calculation domains and strategies are applied. As an example to find a proper external distance for field analysis, a constant length of layer should be kept by adding new layers to the previous one rather than stretching the existing layers. Fig. 5(a) shows that for an external distance of more than $40 \mathrm{~mm}$ in the radial direction, the equivalent self-inductance of winding one, W1 $\left(\mathrm{L}_{11}\right)$ remained almost constant $(\approx 52 \mu \mathrm{H})$ in case of constant length of layers and it presented $15 \%$ error $(\approx 60 \mu \mathrm{H})$ for stretchable elements. Fig. 5(b) presents the computed value of $L_{11}$ for various external distances. One can see that the best selection for the number of layers is 14 and for external distances more than $60 \mathrm{~mm}$, the calculated value of inductance changes less than $5 \%(\approx 2.5 \mu \mathrm{H}$ increase). Selection of a reasonable value of 3-D elements will results in an acceptable computation time and accuracy. For example, in case of considering 14400 elements (72 sector in $\theta$ direction, ten ring in $Z$-direction, and 20 layer in radial direction) the total computation time was about $230 \mathrm{~s}$ using a PC system (CPU: 64 bit/3.1-GHz Intel i5-2400, RAM: $4.00 \mathrm{~GB}$ ). The resulted values of core dimension, number of winding turns, and thickness of insulator are used to implement the MWT prototype as is discussed in the next section. 


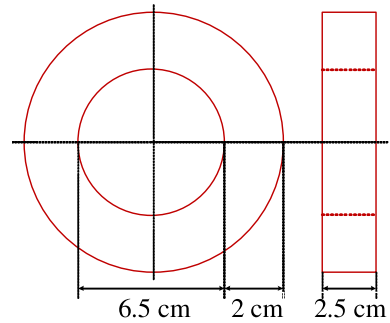

(a)

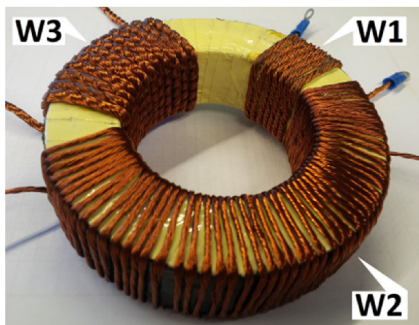

(b)
Fig. 6. (a) Dimensions of magnetic core. (b) Prototype of transformer and windings.

\section{Prototyping of the Transformer}

A prototype of three winding high-frequency toroidal transformer is implemented for a residential microgrid that we are developing. The first stage of the transformer design was selection of magnetic materials because core permeability factor was required for numerical design. The soft ferrite have been widely used in high-frequency converters due to their availability and low price although their saturation flux density is low $(0.3-0.5 \mathrm{~T})$ and results in the transformer large size. Amorphous alloy and nanocrystalline materials have high saturation flux density, high permeability, and low core loss. Comparing their characteristics show that nanocrystalline materials have lower core loss than the amorphous alloys although their saturation flux density $(0.8-1 \mathrm{~T})$ is much lower than that of amorphous alloys (1.4$1.6 \mathrm{~T})$ [11]. The Metglas amorphous alloy 2605SA1 strip of 25 -mm width and $20-\mu \mathrm{m}$ thickness made by Hitachi metals is finally selected taking into account specific core loss, maximum flux density, cost and availability. To develop the magnetic core, the Metglas 2605SA1 thin tape was glued with Araldite 2011 on the surface of each layer and wounded around a cylindrical frame with external diameter of $65 \mathrm{~mm}$. The developed core dimension and the transformer are presented in Fig. 6. The hysteresis loops and the core loss properties were measured experimentally. The excitation waveforms are generated by a TTiTGA1244 programmable waveform generator and are amplified using high-frequency signal amplifier AM3002. A power analyzer PM3000A is employed to measure the transformer power losses and Tektronix current measurement probe TCPA300 and high-voltage differential probe P5200 are used to observe the transformer waveforms as presented in Fig. 7(a). To measure the $B-H$ curve of developed core under $10-\mathrm{kHz}$ square-wave excitation current, winding one is selected as excitation coil and winding three as pickup coil. Considering almost uniform flux density inside the core, the magnetic field intensity $H$ can be calculated using Ampere's law as

$$
H=N_{A} i_{A}(t) / l_{e}
$$

where $N_{A}$ is the number of turns in excitation winding, $i(t)$ is the measured excitation current, and $l_{e}$ is the mean length of the toroidal core. To calculate the magnetic flux density in the core, Faraday's law should be applied using open-circuit voltage of

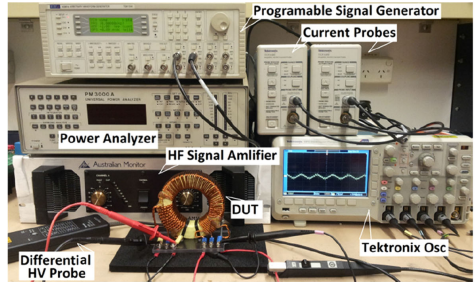

(a)

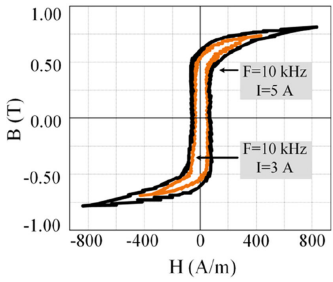

(b)

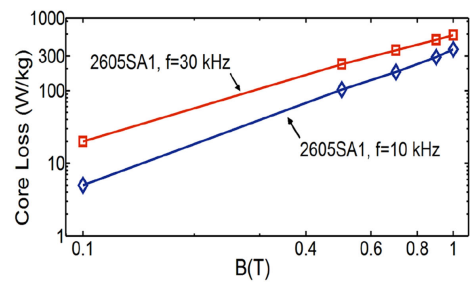

(c)
Fig. 7. (a) Experimental test platform. (b) Measured hysteresis loops the magnetic core for $f=10 \mathrm{kHz}, 3 \mathrm{~A}$, and $5 \mathrm{~A}$. (c) Measured core loss of Metglas 2605SA1 for $10-\mathrm{kHz}$ and $30-\mathrm{kHz}$ excitation currents.

the pickup coil

$$
B=\frac{1}{N_{B} A_{e}} \int V_{B}(t) d t
$$

where $N_{B}$ is the number of turns of pickup coil, $A_{e}$ is the cross-sectional area of magnetic core, and $V_{B}$ is the measured open-circuit voltage of the winding. The recorded measured data are used to plot the $B-H$ loop as shown in Fig. 7(b).

The core loss was measured for two cases of $10-\mathrm{kHz}$ and 30-kHz square-wave excitation currents as shown in Fig. 7(c). The total loss (core loss plus copper loss) was measured using a power analyzer. Considering almost equal ac and dc winding resistances in case of using Litz wires, the copper loss can be calculated easily and the core loss can be defined. The core loss was calculated using the Steinmetz equation as

$$
P_{\text {Coreloss }}=k f^{m} B^{n}
$$

where $f$ is frequency and $B$ is the magnitude of flux density. The resulted core loss characteristics are used to define the new coefficients $(k=6.21, m=1.60, n=1.64)$ for (11) under squarewave currents as the datasheet coefficients $(k=6.5, m=1.51$, $n=1.74$ ) are derived under sinusoidal test currents. To reduce the skin and proximity effects resulted in high-frequency currents, and consequently, reduce the ac resistance and winding loss, a Litz wire should be used for transformer windings. The minimum diameter of a single strand of a Litz wire, $d$ was defined using

$$
d=\sqrt{\frac{4 i}{J \pi S_{n}}}
$$

where $i$ is the winding current, $S_{n}$ is the number of isolated strands, and $J$ is the current density. In our design for all three windings, the Litz wires with diameter of $0.35 \mathrm{~mm}$ are considered. The number of twisted insulated strands for windings one, two, and three is $30(3 * 10), 18(3 * 6)$, and $45(3 * 3 * 5)$ and their number of turns resulted from design process is 7,53 , and 12 turns, respectively. The overall diameters of twisted wires for 
windings were measured approximately as $\mathrm{d}_{1} \approx 3 \mathrm{~mm}, \mathrm{~d}_{2} \approx 2$ $\mathrm{mm}$, and $d_{3} \approx 4 \mathrm{~mm}$. The experimental test desk of Fig. 7(a) was used to test the validity of design process and measure the transformer parameters. The test process is based on the method suggested in [32] due to the MWT high leakage inductance. The programmable signal generator, TTi-TGA1244, and the HF signal amplifier are used to generate the sinusoidal excitation currents. The standard short- and open-circuit tests have been used conventionally to determine the transformer inductive and resistive elements. It has been shown that short-circuit test has less accuracy when the winding resistances and the leakage reactances have significant values relative to the magnetizing impedance [32], [33]. It also will lose its accuracy in case of big difference between the primary and secondary leakage inductances and winding resistances due to winding geometries and configurations. On the other hand, it is necessary to have the same value of magnetic flux, and consequently, permeability factor in the core during all stages of experimental tests. It is difficult to maintain the same magnetizing current in the shortcircuit test because of parallel connection of the magnetizing branch and the secondary winding impedance during the test [32]. Therefore, the short-circuit test did not provide reliable results and was excluded from our test procedures. To measure the transformer leakage and self-inductances, the conventional open circuit and the differentially and cumulatively coupled tests are applied to the windings with one as the primary and three as the secondary. To discuss the experimental test process, windings one and three are selected as examples and the test procedure is applied similarly to other two pairs (windings one and two or windings two and three).

\section{A. Open-Circuit Test}

During this test, the test voltage set as $110 \mathrm{~V}$ at $10 \mathrm{kHz}$ was applied to winding one, while winding three was open circuited. The values of impedances are calculated using the normal equation of sinusoidal exited circuits [32]. The measured impedance including the real and imaginary parts is

$$
\begin{aligned}
Z_{1}(\mathrm{OC}) & =R_{1}(\mathrm{OC})+j \omega L_{1}(\mathrm{OC}) \\
& =\left(R_{W 1}+R_{m}\right)+j \omega\left(L_{l 1}+L_{13}\right) .
\end{aligned}
$$

At the second stage, the excitation current was applied to winding three with the other two windings open circuited [32]. The resultant impedance in this case is

$$
\begin{aligned}
Z_{3}(\mathrm{OC}) & =R_{3}(\mathrm{OC})+j \omega L_{3}(\mathrm{OC}) \\
& =\left(R_{W 3}+R_{m} n^{2}\right)+j \omega\left(L_{l 3}+n^{2} L_{13}\right)
\end{aligned}
$$

where $n$ is the turn ratio of winding three to winding one. The schematics of the test circuits are shown in Fig. 8(a) and (b).

\section{B. Series-Coupling Tests}

In this test, winding one is considered as the primary and winding three as the secondary. They are connected in series in differential or cumulative form as illustrated in Fig. 8(c) and (d), while winding two was open circuited. The series-coupling test is used to factor in the effects on the leakage and magnetizing

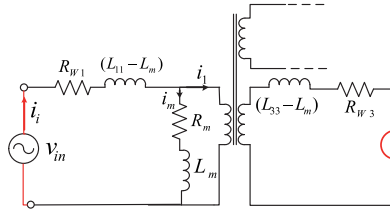

(a)

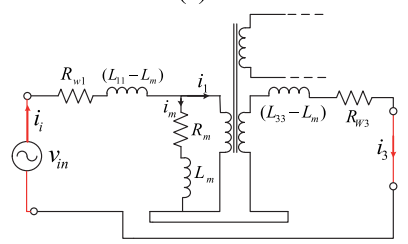

(c)

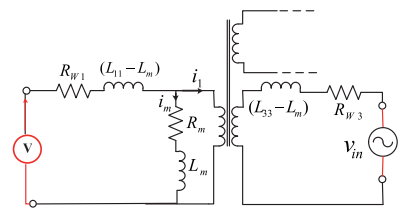

(b)

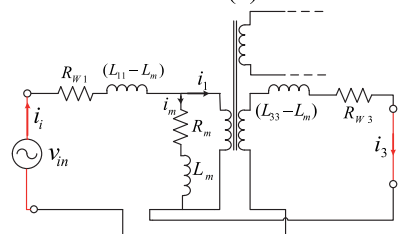

(d)
Fig. 8. Experimental tests of multiwinding transformer. (a) Secondary open-circuit test. (b) Primary open-circuit test. (c) Differentially coupled test. (d) Cumulatively coupled test.

inductances of core excitation [32]. Compared with the parallel coupling test, the measured inductances and resistances are the algebraic addition and subtraction of resistive and inductive elements of the transformer windings, which facilitates direct measurement by the meter. The input impedances measured in differential and cumulatively coupled tests are as ings, which facilitates direct measurement by the meter. The input impedances measured in differential and cumulatively coupled tests are

$$
\begin{aligned}
Z_{1}(\text { dif })= & R_{13}(\text { dif })+j \omega L_{13}(\text { dif }) \\
= & \left(R_{W 1}+R_{W 3}+(1-n)^{2} R_{m}\right) \\
& +j \omega\left(L_{l 1}+L_{l 3}+(1-n)^{2} L_{13}\right) \\
Z_{1}(\mathrm{Cum})= & R_{13}(\text { cum })+j \omega L_{13}(\text { cum }) \\
= & \left(R_{W 1}+R_{W 3}+(1+n)^{2} R_{m}\right) \\
& +j \omega\left(L_{l 1}+L_{l 3}+(1+n)^{2} L_{13}\right) .
\end{aligned}
$$

A combination of series-coupling and the open-circuit tests have been used to find the value of transformer parameters [32]. The resultant equations in terms of inductances $L_{1}(\mathrm{oc})$, $L_{3}$ (oc), $L_{13}$ (dif), and $L_{13}$ (cum) and the resistances $R_{1}$ (oc), $R_{3}$ (oc), $\quad R_{13}$ (dif), and $R_{13}$ (cum) and $R_{m}$ are tabulated in Table I. The resulted values of all three tabulated equations were quite consistent and their average is considered as the final value of the parameter. To guarantee the same value of magnetizing flux density into the core during all the tests, the following relationships should be considered for excitation current during various tests [32]. This will results in a consistent flux density in the core during all tests, and hence, an almost constant values for $L_{m}$ and $R_{m}$.

$$
I_{p(\mathrm{OC})}=n I_{s(\mathrm{OC})}=I_{\mathrm{dif}}(1-n)=I_{\mathrm{cum}}(1+n)
$$

where $I_{P}$ and $I_{\mathrm{s}}$ denote the primary and secondary currents, respectively. It should be noticed that during the series-coupling test the value of $L_{m}$ is equal to the mutual inductance of two windings under the test ( $L_{13}$ in this case). Fig. 9 shows the resultant values of inductances and resistances for windings one and three with the excitation current ranging from 0.5 to $4.5 \mathrm{~A}$. From Fig. 9(a), it is clear that as the winding current increases, the 
TABLE I

Equations of Model Parameters for Different Test Procedures

\begin{tabular}{|c|c|c|c|}
\hline Test Parameter & Differentially Coupled and open-Circuit Tests & Cumulatively Coupled and Open Circuit Tests & Combination of all Three Tests \\
\hline \multirow{2}{*}{$R_{W 1}$} & $(2 n-1) R_{1}(\mathrm{OC})-R_{3}(\mathrm{OC})+R_{13}(\mathrm{dif})$ & $(2 n+1) R_{1}(\mathrm{OC})+R_{3}(\mathrm{OC})-R_{13}(\mathrm{cum})$ & \multirow{2}{*}{$R_{1}(\mathrm{OC})-\frac{R_{13}(\mathrm{cum})-R_{13}(\mathrm{dif})}{4 n}$} \\
\hline & $2 n$ & $2 n$ & \\
\hline \multirow{2}{*}{$R_{W 3}$} & $(2-n) R_{3}(\mathrm{OC})-n R_{1}(\mathrm{OC})+n R_{13}(\mathrm{dif})$ & $(2+n) R_{3}(\mathrm{OC})+n R_{1}(\mathrm{OC})-n R_{13}(\mathrm{cum})$ & \multirow{2}{*}{$R_{3}(\mathrm{OC})-\frac{n R_{13}(\mathrm{cum})-n R_{13}(\mathrm{dif})}{4}$} \\
\hline & 2 & 2 & \\
\hline \multirow{2}{*}{$R_{m}$} & $R_{1}(\mathrm{OC})+R_{3}(\mathrm{OC})-R_{13}(\mathrm{dif})$ & $R_{13}(\mathrm{cum})-R_{1}(\mathrm{OC})-R_{3}(\mathrm{OC})$ & \multirow{2}{*}{$\frac{R_{13}(\mathrm{cum})-R_{13}(\text { dif })}{4 n}$} \\
\hline & $2 n$ & $2 n$ & \\
\hline \multirow{2}{*}{$L_{l 1}$} & $(2 n-1) L_{1}(\mathrm{OC})-L_{3}(\mathrm{OC})+L_{13}(\mathrm{dif})$ & $(2 n+1) L_{1}(\mathrm{OC})+L_{3}(\mathrm{OC})-L_{13}(\mathrm{cum})$ & \multirow{2}{*}{$L_{1}(\mathrm{OC})-\frac{L_{13}(\mathrm{cum})-L_{13}(\mathrm{dif})}{4 n}$} \\
\hline & $2 n$ & $2 n$ & \\
\hline \multirow{2}{*}{$L_{l 3}$} & $(2-n) L_{3}(\mathrm{OC})-n L_{1}(\mathrm{OC})+n L_{13}(\mathrm{dif})$ & $(2+n) L_{3}(\mathrm{OC})+n L_{1}(\mathrm{OC})-n L_{13}(\mathrm{cum})$ & \multirow{2}{*}{$L_{3}(\mathrm{OC})-\frac{n L_{13}(\mathrm{cum})-n L_{13}(\mathrm{dif})}{4}$} \\
\hline & 2 & 2 & \\
\hline \multirow{2}{*}{$L_{13}$} & $L_{1}(\mathrm{OC})-L_{3}(\mathrm{OC})-L_{13}(\mathrm{dif})$ & $L_{13}(\mathrm{cum})-L_{1}(\mathrm{OC})-L_{3}(\mathrm{OC})$ & $L_{13}(\mathrm{cum})-L_{13}($ dif $)$ \\
\hline & $2 n$ & $2 n$ & $4 n$ \\
\hline
\end{tabular}

mutual inductance between windings one and three decreases, while their leakage inductance increases. On the other hand, Fig. 9(b) shows that the value of equivalent core loss resistance will increase with any increase in the test current while the winding resistances are not dependent on the test current and remain constant. To find the accuracy of implementation process, the experimentally measured inductances are compared with the numerically calculated ones. Fig. 9(c) shows the measured values of leakage and self-inductance of winding one with excitation current of 5 A. Comparing measured values with numerically calculated inductances shows that the resulted error is reduced to less than $15 \%$ considering 144003 -D elements for magnetic structure. A further reduction of error to $10 \%$ was achieved by increasing the number of elements to 19440 elements although it increased the computation time considerably (about $11 \mathrm{~min}$ ). The resultant error can be attributed to various experimental factors that cannot be included properly in the numerical analysis, such as stacking factor and windings distribution.

\section{VAlidity TESt of DEsigned TRANSFormeR}

The major parasitic effects are due to the skin effect, proximate effect, and stray capacitance. In this research, the skin and proximity effects are considered in the design process and the effect of stray capacitance is safely ignored because of their small value and relatively low switching frequency, about $10 \mathrm{kHz}$. The numerical model of MWT based on the measured parameters was simulated under different load conditions for medium-frequency range using MATLAB/Simulink. The results are compared with the experimental tests to validate the modeling process. As the windings current in our application are nonsinusoidal including high-frequency harmonics, the se- lected frequency range covers the fundamental frequency of current $(10 \mathrm{kHz})$ and its harmonics $(30,50$, and $70 \mathrm{kHz})$.

The excitation current was applied to winding one of the MWT as the primary and winding three as the secondary where the output signals for entire frequency range were recorded. The experimental tests were carried out for three cases of inductive load, capacitive load, and short-circuit conditions to factor in both mutual and leakage inductive effects. A sinusoidal voltage of $150 \mathrm{~V}(\mathrm{rms})$ with variable frequency changes from 10 to $150 \mathrm{kHz}$ generated by programmable the signal generator and amplified by the HF signal amplifier was used as excitation source. The test process was carried out for other pairs of windings and the results were consistent. Fig. 10(a)-(c) shows the equivalent circuit of windings one and three under the test for inductive load, capacitive load, and short-circuit condition, while winding two is open circuited. The transfer function of circuit in case of capacitive load can be defined as

$$
\begin{aligned}
& \frac{V_{O}(S)}{V_{\text {in }}(S)}= \\
& \frac{Z}{\left[R_{W 1}+\left(L_{11}-L_{m}\right) S+Z\right]\left[1+S C^{\prime} R_{W 3}^{\prime}+S^{2} C^{\prime}\left(L_{33}^{\prime}-L_{m}\right)\right]}
\end{aligned}
$$

where $\mathrm{Z}$ can be found as

$$
[Z]^{-1}=\left[R_{m}+S L_{m}\right]^{-1}+\left[R_{W 3}^{\prime}+S\left(L_{33}^{\prime}-L_{m}\right)+1 / S C^{\prime}\right]^{-1} .
$$

In case of inductive load, the transfer function of secondary to primary voltage and impedance $Z$ can be calculated as (20) and (21), shown at the bottom of the page.

$$
\begin{aligned}
& \frac{V_{O}(S)}{V_{\mathrm{in}}(S)}=\frac{Z^{\prime}\left(r_{O}^{\prime}+L_{O}^{\prime} S\right)}{\left[R_{W 1}+\left(L_{11}-L_{m}\right) S+Z^{\prime}\right]\left[r_{O}^{\prime}+R_{W 3}^{\prime}+S\left(L_{O}^{\prime}+L_{33}^{\prime}-L_{m}\right)\right]} \\
& {\left[Z^{\prime}\right]^{-1}=\left[R_{m}+S L_{m}\right]^{-1}+\left[r_{o}^{\prime}+R_{W 3}^{\prime}+S\left(L_{O}^{\prime}+L_{33}^{\prime}-L_{m}\right)\right]^{-1}}
\end{aligned}
$$




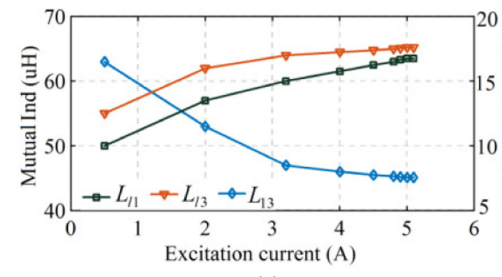

(a)

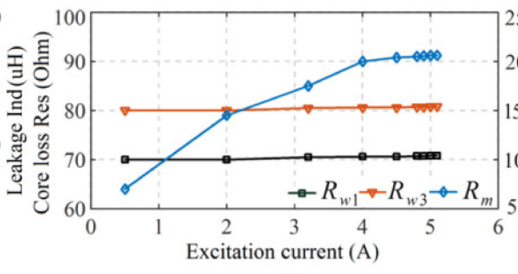

(b)

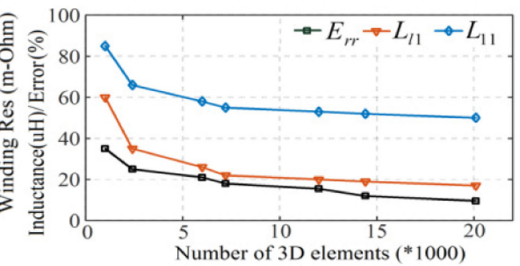

(c)

Fig. 9. Measured values of (a) inductances, (b) resistances for windings one and three, and (c) leakage inductance and self-inductance of winding one and percentage of error versus number of 3-D elements.

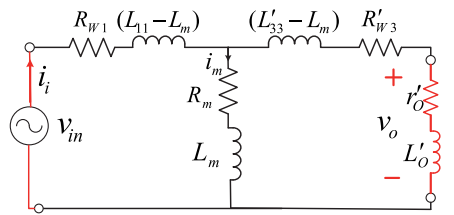

(a)

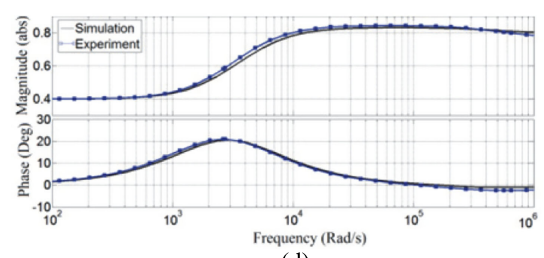

(d)

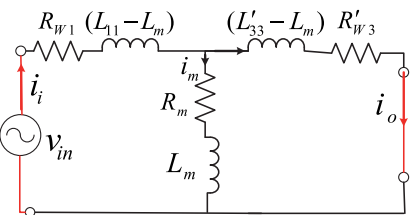

(b)

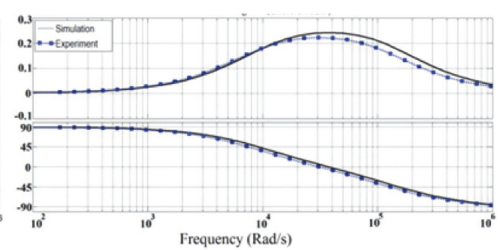

(e)

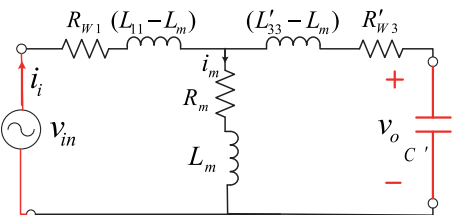

(c)

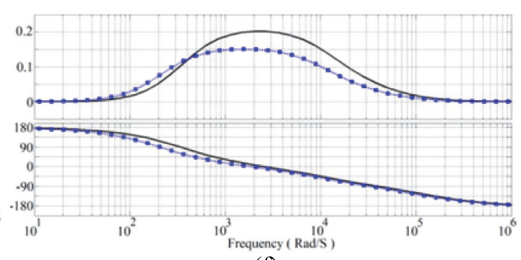

(f)

Fig. 10. Equivalent circuit of MWT under (a) inductive load, (b) short-circuit, and (c) capacitive load test conditions. Frequency response of winding one to three of MWT for cases (d) inductive load, (e) short-circuit, and (f) capacitive load conditions (solid line is simulation and dashed line is experimental results).

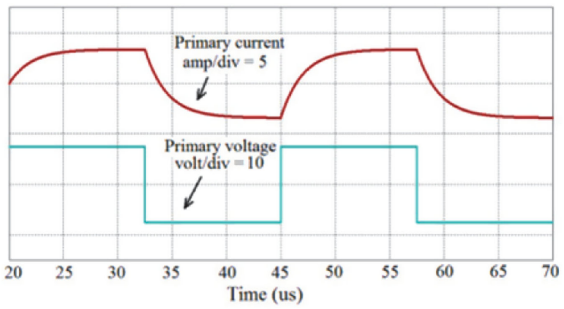

(a)

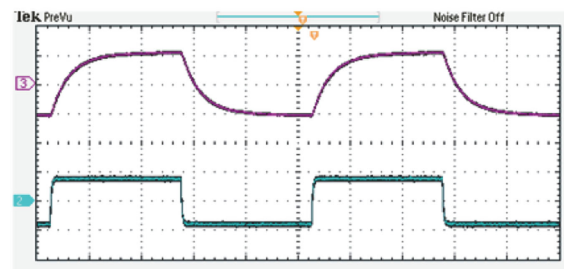

(d)

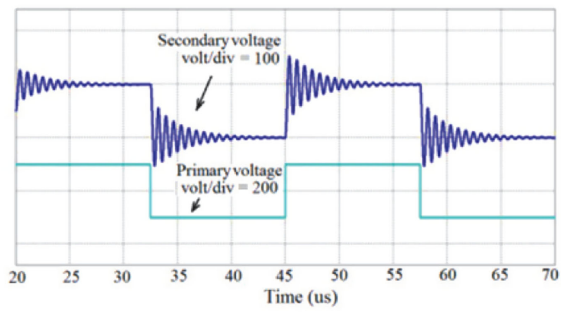

(b)

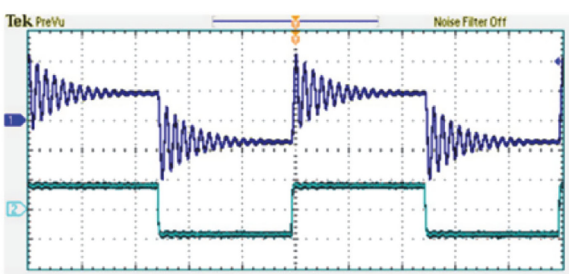

(e)

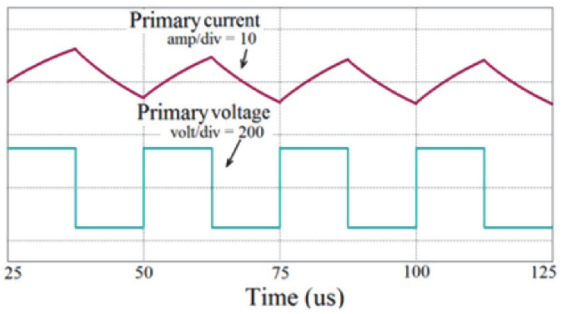

(c)

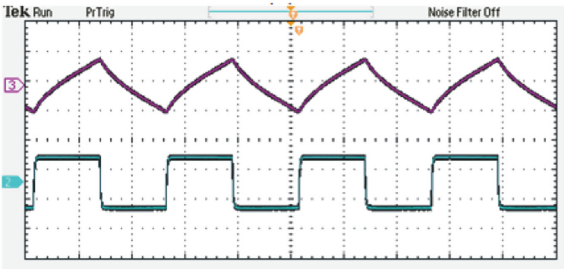

(f)

Fig. 11. Simulation results of MWT model for square-wave excitation using PSIM for cases of (a) short-circuit condition, (b) capacitive load, and (c) inductive load and experimental test results for (d) short-circuit condition, (e) capacitive load, and (f) inductive load conditions, CH1: Secondary voltage, $\mathrm{CH}$ 2: Primary voltage, $\mathrm{CH} 3$ : Primary current.

The last test was carried out under the short-circuit condition and the transfer function and impedance $\mathrm{Z}$ can be defined as

$$
\begin{aligned}
\frac{I_{O}(S)}{V_{\mathrm{in}}(S)} & =\frac{Z}{\left[R_{W 1}+\left(L_{11}-L_{m}\right) S+Z\right]\left[R_{W 3}^{\prime}+S\left(L_{33}^{\prime}-L_{m}\right)\right]} \\
{[Z]^{-1} } & =\left[R_{m}+S L_{m}\right]^{-1}+\left[R_{W 3}^{\prime}+S\left(L_{33}^{\prime}-L_{m}\right)\right]^{-1} .
\end{aligned}
$$

Comparing experimental and simulation results shows that the simulated model adapts very well with experimental test results for all three types of loads. Looking at Fig. 10(d)-(f) shows that the difference between the simulated and experimental results is small for medium-frequency range as the modeling process and experimental tests are carried out for this range. The measured steady-state core and the winding temperatures were in the range of $40-60{ }^{\circ} \mathrm{C}$ during the frequency response tests 
under the nominal load conditions. Within this temperature range the effect of temperature on winding resistance is negligible. On the other hand the magnetic characteristics of amorphous core material, used for the transformer are almost independent to temperature according to the data provided by manufacturer, therefore, the effect of temperature on the transformer parameters were negligible. Fig. 11 compares the experimental and simulation results in the time domain with a $40-\mathrm{kHz}$ square-wave excitation. It can be seen that the simulations and experimental wave forms show a very good agreement for all three types of load conditions. Based on the results the suggested method for design and characterization of MWTs is validate for mediumfrequency range where parasitic effect of stray capacitors are negligible and the operation point of magnetic component is in linear area of $B-H$ curve.

\section{CONCLUSION}

Due to the application of MWTs as a common magnetic link in integration of renewable energy sources, they have attracted considerable research interest. This paper covered three stages of design, prototyping, and experimental test of a high-frequency toroidal MWT. The RNM was used to design the transformer based on the required specifications due to the low computation time. A prototype transformer was developed using amorphous magnetic materials and the transformer parameters including leakage and mutual inductances and resistances are measured applying open-circuit and series-coupling tests. A comparison between the simulation and experimental test results under different loads within the medium-frequency range validated both design and modeling procedures.

\section{REFERENCES}

[1] D. Gunasekaran and L. Umanand, "Integrated magnetics based multi-port bidirectional DC-DC converter topology for discontinuous-mode operation," IET Power. Electron., vol. 5, no. 7, pp. 935-944, Aug. 2012.

[2] M. R. Islam, Y. G. Guo, Z. W. Lin, and J. G. Zhu, "An amorphous alloy core medium frequency magnetic-link for medium voltage PV inverters," J. Appl. Phys., vol. 115, no. 17, pp. 17E710-17E710-3, May 2014.

[3] M. Ishigaki, K. Ito, S. Tomura, and T. Umeno, "A new isolated multi-port converter using interleaving and magnetic coupling inductor technologies," in Proc. 28th Annu. IEEE Appl. Power Electron Conf. Expo., Long Beach, CA, USA, 2013, pp. 1068-1074.

[4] Y. M. Chen, Y. C. Liu, and F. Y. Wu, "Multi-input DC/DC converter based on the multiwinding transformer for renewable energy applications," IEEE Trans. Ind. Appl., vol. 38, no. 4, pp. 1096-1104, Jul./Aug. 2002.

[5] M. R. Islam, G. Lei, Y. Guo, and J.Zhu, "Optimal design of high-frequency magnetic links for power converters used in grid-connected renewable energy systems," IEEE Trans. Magn., vol. 50, no. 11, pp. 1-4, Nov. 2014.

[6] I. O. Lee, "Hybrid DC-DC converter with phase-shift or frequency modulation for NEV battery charger," IEEE Trans. Ind. Electron., vol. 63, no. 2, pp. 884-893, Feb. 2016.

[7] J. Zhang, H. Wu, X. Qin, and Y. Xing, "PWM plus secondary-side phase-shift controlled soft-switching full-bridge three-port converter for renewable power systems," IEEE Trans. Ind. Electron., vol. 62, no. 11, pp. 7061-7072, Nov. 2015.

[8] Y. Shi, R. Li, Y. Xue, and H. Li, "Optimized operation of current-fed dual active bridge DC-DC converter for PV applications," IEEE Trans. Ind. Electron., vol. 62, no. 11, pp. 6986-6995, Nov. 2015.

[9] H. Tao, J. L. Duarte, and M. A. M. Hendrix, "Three-port triple-half-bridge bidirectional converter with zero-voltage switching," IEEE Trans. Power Electron., vol. 23, no. 2, pp. 782-792, Mar. 2008.

[10] H. Tao, A. Kotsopoulos, J. L. Duarte, and M. A. M. Hendrix, "Family of multiport bidirectional DC-DC converters," Proc. Inst. Elect. Eng., vol. 153, no. 3, pp. 451-458, 2006.
[11] M. R. Islam, Y. G. Guo, and J. G. Zhu, "High-frequency link multilevel cascaded medium-voltage converter for direct grid integration of renewable energy systems," IEEE Trans. Power Electron., vol. 29, no. 8, pp. 4167-4182, Aug. 2014.

[12] C. Zhao and J. W. Kolar, "A novel three-phase three-port UPS employing a single high-frequency isolation transformer," in Proc. 35th IEEE Power Electron. Spec. Conf., vol. 6, 2004, pp. 4135-4141.

[13] F. de León, S. Purushothaman, and L. Qaseer, "Leakage inductance design of toroidal transformers by sector winding," IEEE Trans. Power Electron., vol. 29, no. 1, pp. 473-480, Jan. 2014.

[14] I. Hernandez, F. de Leon, and P. Gomez, "Design formulas for the leakage inductance of toroidal distribution transformers," IEEE Trans. Power Del., vol. 26, no. 4, pp. 2197-2204, Oct. 2011.

[15] A. J. Binnie and T. R. Foord, "Leakage inductance and interwinding capacitance in toroidal ratio transformers," IEEE Trans. Instrum. Meas., vol. 16, no. 4, pp. 307-314, Dec. 1967.

[16] W. G. Hurley and D. J. Wilcox, "Calculation of leakage inductance in transformer windings," IEEE Trans. Power Electron., vol. 9, no. 1, pp. 121-126, Jan. 1994.

[17] A. Dauhajre and R. D. Middlebrook, "Modelling and estimation of leakage phenomena in magnetic circuits," in Proc. 17th Annu. IEEE Power Electron. Spec. Conf., Vancouver, Canada, 1986, pp. 213-226.

[18] J. Wang, A. F. Witulski, J. L. Vollin, T. K. Phelps, and G. I. Cardwell, "Derivation, calculation and measurement of parameters for a multiwinding transformer electrical model," in Proc. 14th Appl. Power Electron. Conf. Expo., Dallas, TX, USA, 1999, vol. 1, pp. 220-226.

[19] G. Skutt, F. C. Lee, R. Ridley, and D. Nicol, "Leakage inductance and termination effects in a high-power planar magnetic structure," in Proc. 9th Annu. Appl. Power Elec. Conf. Expo., vol. 1, Orlando, FL, USA, 1994, pp. 295-301

[20] H. Tao, A. Kotsopoulos, J. L. Duarte, and M. A. M. Hendrix, "Multiinput bidirectional DC-DC converter combining DC-link and magneticcoupling for fuel cell systems," in Proc. Ind. Appl. Conf., 2005, vol. 3, pp. 2021-2028.

[21] C. Zhao, S. D. Round, and J. W. Kolar, "An isolated three-port bidirectional DC-DC converter with decoupled power flow management," IEEE Trans. Power Electron., vol. 23, no. 5, pp. 2443-2453, Sep. 2008.

[22] H. Tao, A. Kotsopoulos, J. L. Duarte, and M. A. M. Hendrix, "A softswitched three-port bidirectional converter for fuel cell and supercapacitor applications," in Proc. 36th IEEE Power Electron. Spec. Conf., Recife, Brazil, 2005, pp. 2487-2493.

[23] H. Tao, A. Kotsopoulos, J. L. Duarte, and M. A. M. Hendrix, "Triplehalf-bridge bidirectional converter controlled by phase shift and PWM,' in Proc. 21st Annu. IEEE Appl. Power Electron. Conf. Expo., Dallas, TX, USA, 2006, pp. 1256-1262.

[24] J. Worotynski, M. Turowski, and E. Worotynska, "Generation of an optimal reluctance network model for fast 2-D and 3-D simulations of electromagnetic devices," in Proc. 2nd Int. Conf. Comput. Electromagn., 1994, London, U.K., pp. 116-119.

[25] F. Janet, J. L. Coulomb, C. Chillet, and P. Mas, "Magnetic moment and reluctance network mixed method applied to transformer modeling," IEEE Trans. Magn., vol. 41, no. 5, pp. 1428-1431, May 2005.

[26] D. Vanoost, H. De Gersem, J. Peuteman, G. Gielen, and D. Pissoort, "Twodimensional magnetostatic finite-element simulation for devices with a radial symmetry," IEEE Trans. Magn., vol. 50, no. 5, pp. 1-4, May 2014.

[27] B. Auchmann, B. Flemisch, and S. Kurz, "A discrete 2-D formulation for 3-D field problems with continuous symmetry," IEEE Trans. Magn., vol. 46, no. 8, pp. 3508-3511, Aug. 2010.

[28] Z. Zhanxin, X. Dexin, W. Gang, Z. Yanli, and Y. Xiuke, "Computation of 3-D magnetic leakage field and stray losses in large power transformer," IEEE Trans. Magn., vol. 48, no. 2, pp. 739-742, Feb. 2012.

[29] S. Ghalavand, V. Zadeh, and Isfahani, "An improved magnetic equivalent circuit model for iron-core linear permanent-magnet synchronous motors," IEEE Trans. Magn., vol. 46, no. 1, pp. 112-120, Jan. 2010.

[30] M. Amrhein and P. T. Krein, "3-D magnetic equivalent circuit framework for modeling electromechanical devices," IEEE Trans. Energy Convers., vol. 24, no. 2, pp. 397-405, Jun. 2009.

[31] S. V. Kulkarni and S. A. Khaparde, Transformer Engineering Design \& Practice. New York, NY, USA: Marcel Dekker, 2004.

[32] J. G. Hayes, D. Cashman, M. G. Egan, and T. O’Donnell, "Comparison of test methods for characterization of high-leakage two-winding transformers," IEEE Trans. Ind. Appl. vol. 45, no. 5, pp. 1729-1741, Sep./Oct. 2009.

[33] J. G. Hayes, N. O’Donovan, M. G. Egan, and T. O’Donnell, "Inductance characterization of high-leakage transformers," in Proc. IEEE Appl. Power Electron. Conf., 2003, pp. 1150-1156. 


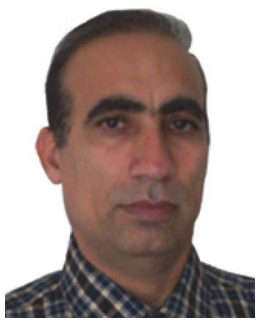

Mohammad Jafari (M'12) received the B.Sc. degree from Shiraz University, Shiraz, Iran, in 1998, and the M.Sc. degree from Guilan University, Rasht, Iran, in 2001, both in electrical engineering. He is currently working toward the Ph.D. degree at the University of Technology Sydney (UTS), Sydney, Australia.

From 2001 to 2011 , he contributed to the design and development of industrial power electronic projects. He has contributed to the design and development of many industrial power electronic projects. Since 2012, he has been with the UTS, as a Lecturer His current research interests include power electronic converters, renewable energy systems, and smart grids.

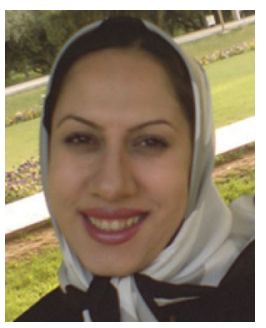

Zahra Malekjamshidi (S'13) received the B.Sc. and M.Sc. degrees from Shiraz University, Shiraz, Iran, in 1998, and 2001, respectively, both in electrical engineering. She is currently working toward the Ph.D. degree at the University of Technology Sydney, Sydney, Australia.

Her research interests include matrix converters, dc-dc converters, renewable energy technologies, and smart grids.

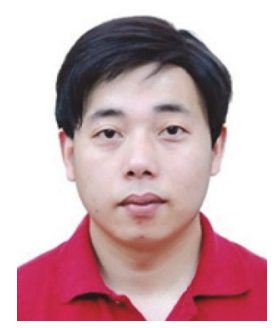

Gang Lei (M'14) received the B.S. degree in mathematics from Huanggang Normal University, Huanggang, China, in 2003, and the M.S. degree in mathematics and the Ph.D. degree in electrical engineering from Huazhong University of Science and Technology, Wuhan, China, in 2006 and 2009, respectively.

$\mathrm{He}$ is currently a Lecturer at the University of Technology Sydney, Sydney, Australia. His current research interests include electromagnetic inverse problems, design optimization of electri-

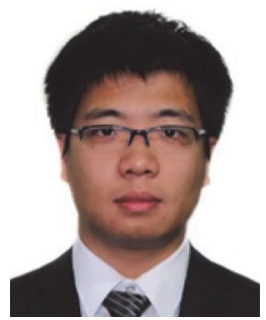

Tianshi Wang received the B.E. degree from Harbin Institute of Technology, Harbin, China, in 2008 , and the M.E. degree from the University of Technology Sydney, Sydney, Australia, in 2013, where he is currently working toward the Ph.D. degree.

His research interests include design, optimization, and advanced control algorithms of ac motor drives, optimal pulse width modulation, and advanced digital control with real-time implementation.

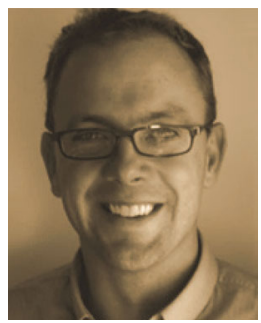

Glenn Platt received the Ph.D. degree from the University of Newcastle, Newcastle, Australia.

He leads the Grids and Energy Efficiency program within CSIRO's Energy Flagship, Newcastle, focusing on solving the energy challenges of consumers, business, and grids. The program's work ranges from energy storage, electric vehicles, smart grids, and the integration of largescale solar systems, through to solar cooling, energy management systems, economics and understanding people's response, and uptake of particular low-carbon energy options.

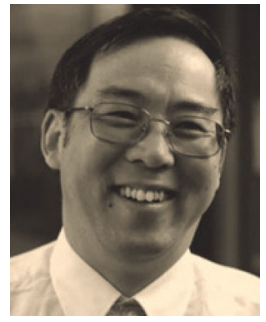

Jianguo Zhu (S'93-M'96-SM'03) received the B.E. degree from Jiangsu Institute of Technology, Jiangsu, China, in 1982, the M.E. degree from Shanghai University of Technology, Shanghai, China, in 1987, and the Ph.D. degree from the University of Technology Sydney (UTS), Sydney, Australia, in 1995.

$\mathrm{He}$ is currently a Professor of electrical engineering and the Head of the School of Electrical, Mechanical and Mechatronic Systems, UTS. His research interests include electromagnetics, magnetic properties of materials, electrical machines and drives, power electronics, and renewable energy systems. 


\title{
Design and Implementation of an Amorphous High-Frequency Transformer Coupling Multiple Converters in a Smart Microgrid
}

\author{
Mohammad Jafari, Member, IEEE, Zahra Malekjamshidi, Student Member, IEEE, \\ Gang Lei, Member, IEEE, Tianshi Wang, Glenn Platt, and Jianguo Zhu, Senior Member, IEEE
}

\begin{abstract}
Recent improvements in magnetic material characteristics and switching devices have generated a possibility to replace the electrical buses with highfrequency magnetic links in microgrids. Multiwinding transformers (MWTs) as magnetic links can effectively reduce the number of conversion stages of renewable energy system by adjusting turn ratio of windings according to the source voltage level. Other advantages are galvanic isolation, bidirectional power flow capability, and simultaneous power transfer between multiple ports. Despite the benefits, design, and characterization of MWTs are relatively complex due to their structural complexity and cross-coupling effects. This paper presents all stages of numerical design, prototyping, and characterization of an MWT for microgrid application. To design the transformer for certain value of parameters, the reluctance network method is employed. Due to the iterative nature of transformer design, it presented less computation time and reasonable accuracy. A prototype of designed transformer is implemented using amorphous magnetic materials. A set of experimental tests are conducted to measure the magnetic characteristics of the core and series coupling and open-circuit tests are applied to measure the transformer parameters. A comparison between the simulation and experimental test results under different loads within the medium-frequency range validated both design and modeling procedures.
\end{abstract}

Index Terms-Design, modeling, multiwinding, reluctance network method (RNM), smart microgrid, transformer.

\section{INTRODUCTION}

$\mathbf{R}$ ECENT advancements on smart microgrid technologies have imposed increasing demands for more reliable and flexible converters and control techniques. In contrast to the traditional electrical ac and dc buses, the high-frequency magnetic links can reduce effectively the number of conversion stages in microgrids with the help of modern soft magnetic materials with

Manuscript received January 29, 2016; revised April 4, 2016; accepted April 28, 2016.

M. Jafari, Z. Malekjamshidi, G. Lei, T. Wang, and J. G. Zhu are with the School of Electrical, Mechanical and Mechatronic Systems, University of Technology Sydney, Sydney, NSW 2007, Australia (e-mail: Mohammad.Jafari@uts.edu.au; Z.Malekjamshidi@ student.uts.edu.au; Gang.Lei@uts.edu.au; Tianshi.Wang@student.uts.edu.au; Jianguo. Zhu@uts.edu.au).

G. Platt is with the Commonwealth Scientific and Industrial Research Organisation (CSIRO), Newcastle, NSW 2304, Australia (e-mail: Glenn.Platt@csiro.au). superior magnetic characteristics and fast- and low-power loss switching devices [1]-[5]. Multiwinding transformers (MWTs) can provide a common magnetic bus for integrating renewable energies in the form of magnetic flux. Their application in the multiactive bridge phase-shift converter makes it possible to simply integrate the sources of different voltage levels using different turn ratios [6]-[9]. Other advantages are galvanic isolation, bidirectional power flow capability, faster control, and simultaneous power transfer among the ports [10]-[12]. Design of MWTs for certain value of inductances is relatively complex due to their complex structureand cross-coupling effects [13]-[15]. Research on MWTs mainly is focused on their characterization and modeling and there is not much research on design methods [16]-[19]. This paper provides a complete discussion on design, prototyping, and experimental tests of a high-frequency toroidal MWT. The transformer is designed for certain values of inductances, using reluctance network method (RNM). A prototype has been fabricated using amorphous magnetic materials to validate the accuracy of proposed design method. To measure the transformer parameters and extract the equivalent electrical model, the open circuit, differential and cumulatively coupled tests are conducted on the prototype transformer. The short-circuit test is excluded as it did not provide reliable results due to relatively high-leakage inductances. The prototype transformer is tested for a wide-frequency range under different load conditions and the results are compared with the simulation based on the extracted transformer model.

\section{MultiWinding Transformer as Common Magnetic LINK}

MWTs have been used as the common magnetic links in multiactive bridge phase-shift converters to integrate the renewable energies effectively [20]-[23]. The converter designed in this research includes four ports connected to the load, fuel cell, battery, and photovoltaic (PV) as illustrated in Fig. 1. The Hbridge units produce high-frequency ac square wave from dc buses linked to the dc sources. The power flow between the ports one, two, and three is controlled by using the phase-shift technique. To apply the technique, port one is selected as the reference and ports two and three are shifted for a leading or lagging phase angle to send or receive power to port one. A dutycycle control is applied to port three for the maximum power point tracking of PV panel. As illustrated in the figure, port one is a bidirectional port transferring power from renewable 


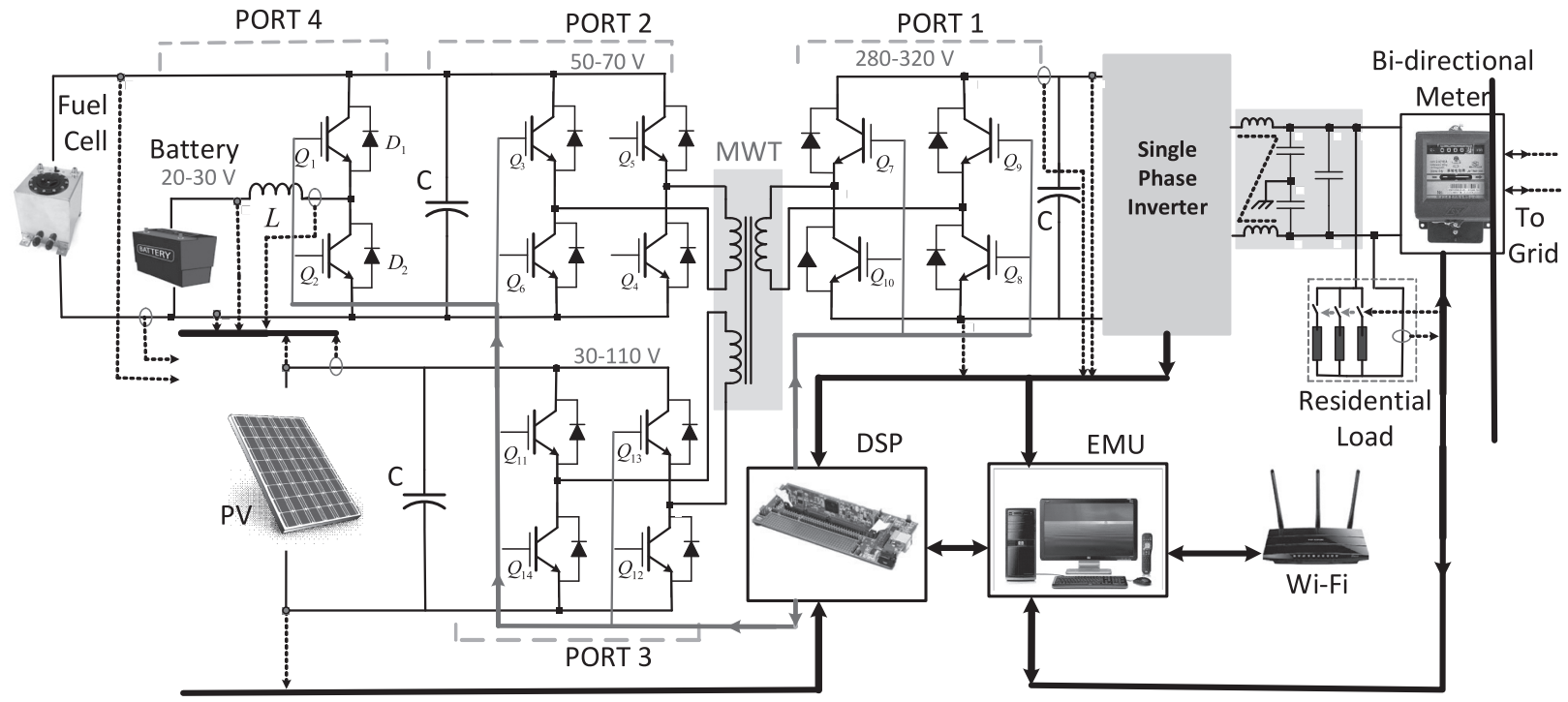

Fig. 1. Structure of proposed multiactive bridge phase-shift converter.

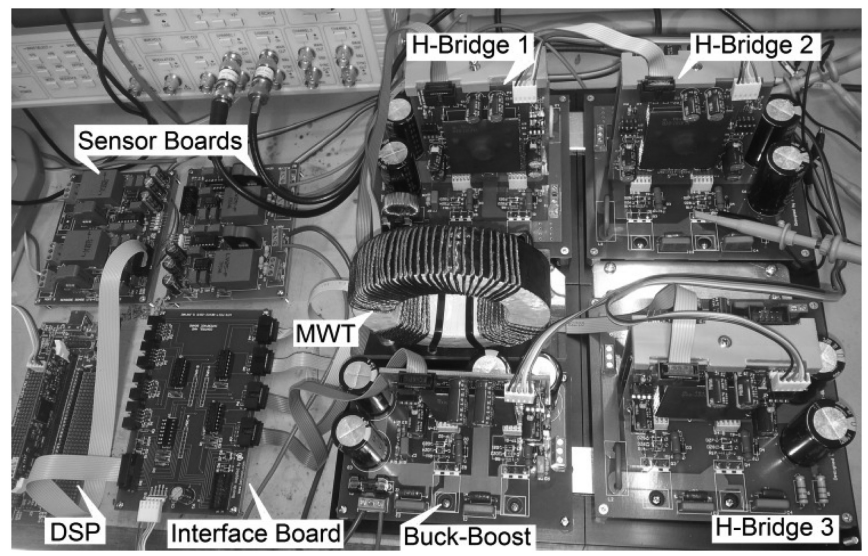

Fig. 2. Experimental set of designed renewable energy system.

sources or battery to the inverter and further to the load and grid. It can also transfer the power reversely from the grid to the battery (using port two and four). Port two is connected to the dc bus linking directly to the fuel cell and a bidirectional buck-boost converter linked to the $24-\mathrm{V}$ battery bank. The voltage of the dc bus is $50-70 \mathrm{~V}$ as it is connected directly to the fuel cell stack. It operates in the buck and boost modes to charge and discharge the battery, respectively. Fuel cell in this system can be used as back up energy source and in normal operation mode, power transfers from PV (port three) as preferred source to the inverter and load (port one). Battery can be charged by fuel cell, PV, or grid and also is used to balance the voltage of $\mathrm{dc}$ bus due to low dynamic response of fuel cell during fuel cell operation. The experimental set of designed renewable energy system including MWT is presented in Fig. 2. The MWT as a common magnetic link provides flexibility in the direction of power flow between the ports. It facilitates the operation of system in different modes based on direction of power flow and applying various energy management scenarios in both gridconnected and islanding modes. The leakage inductances of the transformer windings are used as energy transfer components in this topology [7]-[12]. Using external bulked inductors in series with transformer windings is suggested although it increases the system size and cost [9], [10]. To avoid this problem, the MWT should be designed for certain value of inductances according to the desired range of operating phase shift at the required power throughput. Detail of the converter operation modes is out of scope of this paper as this paper mainly focuses on the design, modeling, and experimental test of the high-frequency MWT.

\section{Design Of MUltiWinding TRANSFORMER}

Design of MWTs for certain value of inductances using the classical methods of transformer design is not accurate due to their structural complexity and cross-coupling effects. Numerical methods such as 2-D and 3-D finite-element methods (FEMs) or RNM are commonly used in design of magnetic structures such as electrical motors and transformers. The FEM is an accurate field analysis method but when used in design optimization, the computational cost is too high. On the other hand, the RNM is very fast but less accurate [24]-[29]. Magnetic field analysis using an FEM can take into account the nonlinearity of magnetic materials, geometry, and actual winding distribution while an RNM is based on linear assumptions. The RNM also can be used for analysis of devices with arbitrary geometries and excitations using a general 3-D element and variable size reluctance network [30]. In this research, the RNM is used in the design process. Due to iterative nature of the transformer design, it presented less computation time and reasonable accuracy. To use it in design optimization, various methods of choosing proper calculation domains such as number of 3-D elements and dimension of field analysis area have been implemented to improve its accuracy. The required values of leakage and self-inductances of transformer windings are selected as design specifications. The number of winding turns, dimension of magnetic core and thickness of insulator between each winding and the core are selected as design variables. To reduce the total time of design, initial values of design variables are defined using classical methods of the transformer design [31]. To find the initial size of the core, the area-product was defined based on the power handling 


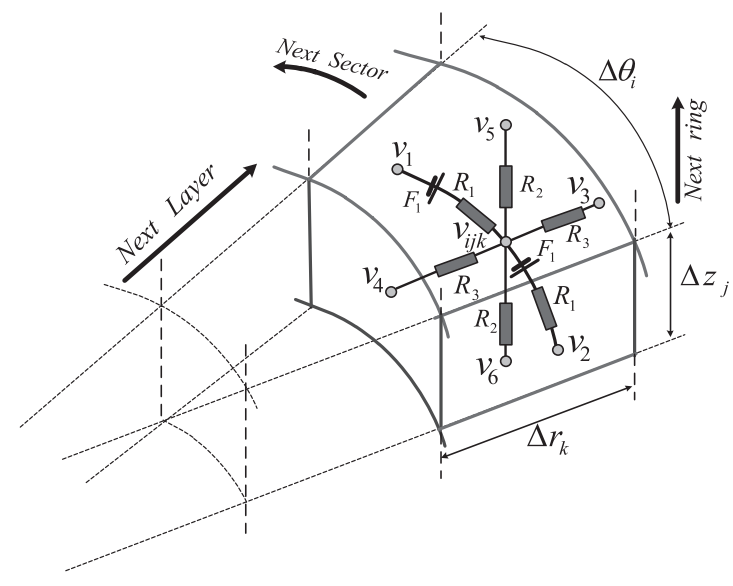

Fig. 3. 3-D reluctance element.

capacity, current density, and flux density. The resultant value then was used to find the core size from area-product/core-size charts. Finally the number of turns was calculated using Faraday's law. To analyze the magnetic field using an RNM, the magnetic structure is divided into three different areas including magnetic core, insulator, and free space around the core. To adjust the leakage inductance of transformer, the thickness of the insulator between windings and the magnetic core considering isolation constraints are selected as variables. To do the analysis, the magnetic structure is assumed to operate in linear section of the magnetization curve, and therefore, a constant value of permeability is used. The magnitude of terminal voltage is chosen such that the peak flux density would just reach the knee point of the magnetization curve, and thus, the linear model can give sufficient accuracy. This is particularly true for the magnetic material used in this design (amorphous 2605SAl) based on data provided by factory and experimental tests presented in Section IV. This can effectively avoid the extra current harmonics and excessive magnetization current and core losses due to the distortion of magnetic field caused by the nonlinear magnetization curve. On the other hand, because of the full use of the linear region, there is no need to use bigger core or more winding than necessary. This assumption also helps to represent the magnetic behavior of the core under nonsinusoidal excitation currents applying the superposition rule to Fourier's terms of the current. Due to the axial symmetry of the toroidal core, the cylindrical coordinate system has been selected for numerical modeling. The core is partitioned into thousands of 3-D elements. Each element includes six reluctances in basic directions of cylindrical coordination, $r, \theta$, and $Z$, and is presented by $Q_{i j k}$.The indexes $i, j$, and $k$ represent the unique situation of 3-D element among the entire magnetic structure. All six branches are connected to a central node at one side and to the boundary nodes at boundary surfaces of 3-D element at the other side as shown in Fig. 3. The reluctances are calculated using geometrical shape and permeability factor of the element and sources, $F_{i j k}$, are representative of magnetic motive force, $m m f$, in the corresponding direction.

The sources should be equal to zero in directions with no magnetic flux, which makes the element simpler for analysis.
The magnetic structure will be divided into thousands of such elements arranged in sectors in $\theta$, layers in radial $r$, and rings in $Z$-directions. The value of voltage sources, $F_{i j k}$ in case of toroidal core in directions of $r$ and $Z$ is almost zero and in $\theta$ direction depends on the winding turns covering the element and the current flowing through it. Applying the nodal analysis to the central node of elements results a group of equations for the central node voltages as unknowns. The final equation of entire magnetic structure considering the equations of all nodes can be written in matrix form as

$$
Y^{\prime} \tilde{V}-Y \tilde{F}=0
$$

where $Y^{\prime}, Y, \tilde{V}$, and $\tilde{F}$ are central-node-linked conductance, source-linked conductance, central-node voltage, and source voltage matrices, respectively. To solve the aforementioned equation and define the potential of all central nodes of the 3-D elements an initial condition is considered. The potential of the central nodes of the elements situated on the last layer and rings are assumed to be zero. The number of nodes and the number of 3-D elements depend on the number of selected divisions in three dimensions of cylindrical coordination. The value of current between the nodes representing the magnetic flux can be calculated using the potential of central nodes of adjacent elements and their interconnected resistances. Considering all branches of the element in three directions, one can calculate the field intensity $H_{i}$, the branch volume $V_{i}$, and the flux density $B_{i}$ for $i=1,2, \ldots, 6$ as [30]

$$
\begin{aligned}
H_{i} & =\left(v_{i j k}-v_{i}\right) / l_{i} \\
V_{i} & =A_{i} l_{i} \\
B_{i} & =\varphi_{i} / A_{i} .
\end{aligned}
$$

The closed-form volumetric integration of the distributed stored energy can be calculated from

$$
W_{S}=\int_{V} \frac{H \cdot B}{2} d V=\frac{\mu}{2} \int_{V}|H|^{2} d V
$$

where $W_{S}$ is the stored energy in a 3-D element enclosed by volume $V$, and $\mu$ the magnetic permeability [30]. The net magnetic energy stored in the $i$ th branch of the element $Q_{i j k}$ based on (2)-(5) assuming constant flux density in the element can be calculated using the following expression:

$$
W_{i}=\left(\mu V_{i} H_{i}^{2}\right) / 2
$$

substituting (2)-(4) into (6) results in

$$
W_{i}=\left(v_{i j k}-v_{1 i j k}-F_{i}\right)^{2} / 2 R_{i}
$$

where $R_{i}$ is the reluctance of the respected branch and the value $F_{i}$ should be equal to zero for branches without $m m f$. The overall energy of a 3-D element $Q_{i j k}$, can be obtained by integrating of the energy in all six branches. To calculate the winding self-inductances, a closed surface enclosing entire magnetic structure is assumed. The total enclosed energy is calculated by integrating the energy of all $n$ elements inside the surface. The resultant total energy, $W_{S}$, can be used to define the equivalent inductance, $L_{\text {eq }}$, using

$$
L_{\mathrm{eq}}=2 W_{s} / I_{s}^{2} .
$$




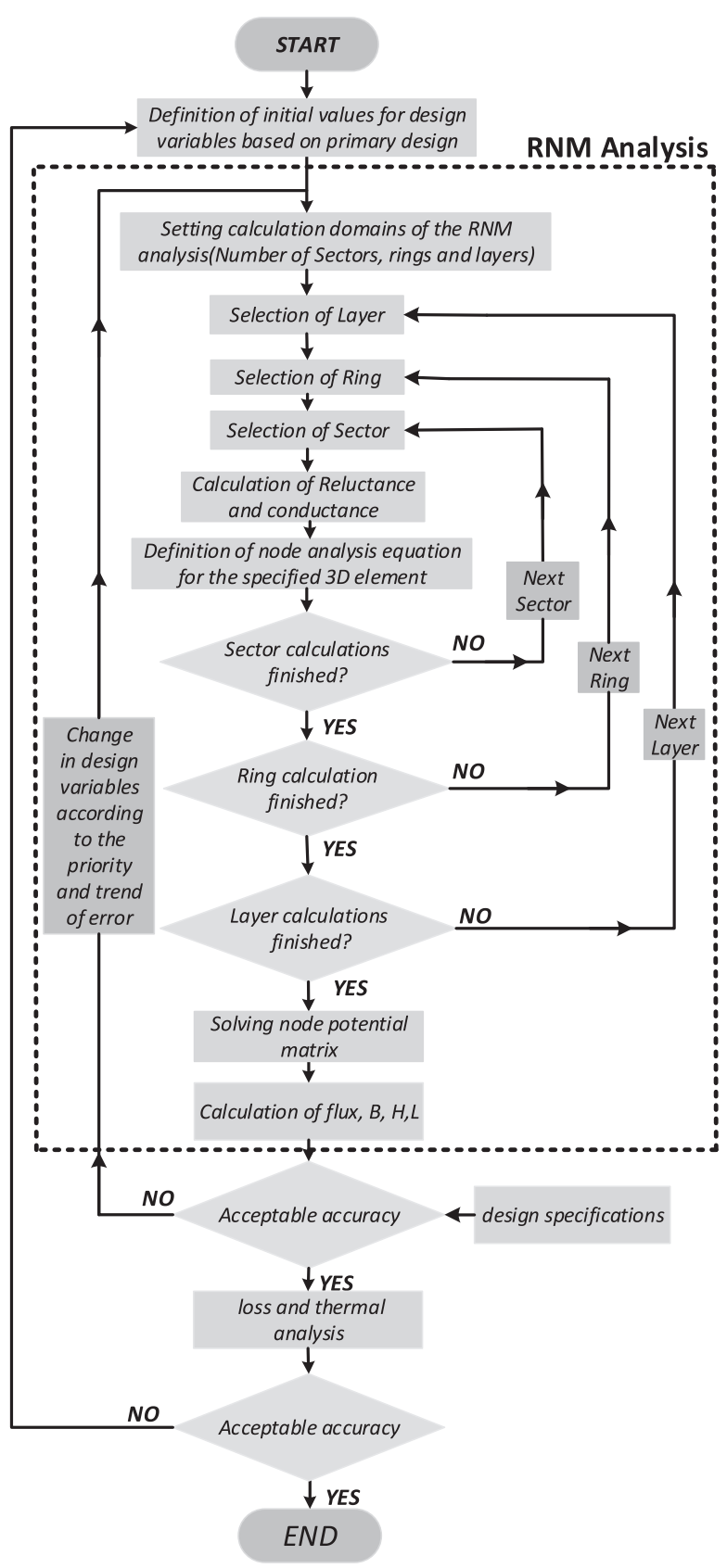

Fig. 4. Flow chart of transformer design process using MATLAB.

The self-inductance of windings one $L_{11}$, two $L_{22}$, and three $L_{33}$ was calculated based on (8). To define the leakage inductances of the windings, $L_{l 1}, L_{l 2}$, and $L_{l 3}$, two different enclosed surfaces, one includes only the core area and the other enclosed core, insulator and surrounding areas (including leakage flux distribution area) are considered and the difference between the energies stored within the volumes enclosed by these surfaces can be used. The values of self- and the leakage inductances of all windings of the MWT are calculated by using the method outlined previously. The core loss resistance for the resultant structure can be found using the Steinmetz equation based on the flux density and frequency. Fig. 4 presents the flow chart showing stages of analysis using MATLAB. As can be seen in

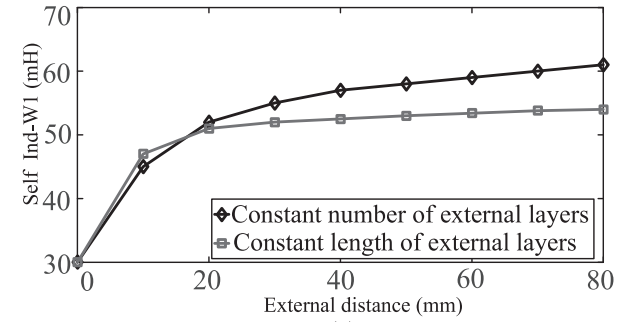

(a)

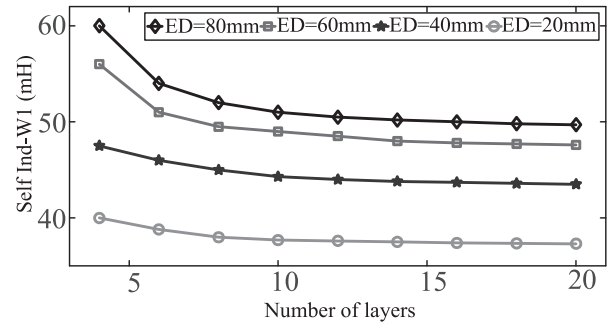

(b)

Fig. 5. (a) Self-inductance of winding one for two cases of fixed and stretchable layer selection. (b) Self-inductance of winding one for various external distances.

the figure, the parts enclosed by dashed line are steps of RNM analysis. The calculation domains such as number of sectors, rings, and layers are defined at first step. The analysis starts with the first sector of the first ring of the first layer and the next ring is selected when all sectors are analyzed and the next layer is selected when analysis of all rings of the previous layer is completed. The value of $B, H$, and $L$ are calculated based on (2)(8). The resultant values are compared with specifications, and in case of excessive error, the design variables are changed according to the priority. In case of acceptable results, the magnetic structure is analyzed for losses and thermal considerations. The loss analysis was carried out based on the Steinmetz equation. To calculate the copper losses, both skin and proximity effects are considered. To improve the accuracy analysis, various calculation domains and strategies are applied. As an example to find a proper external distance for field analysis, a constant length of layer should be kept by adding new layers to the previous one rather than stretching the existing layers. Fig. 5(a) shows that for an external distance of more than $40 \mathrm{~mm}$ in the radial direction, the equivalent self-inductance of winding one, W1 $\left(\mathrm{L}_{11}\right)$ remained almost constant $(\approx 52 \mu \mathrm{H})$ in case of constant length of layers and it presented $15 \%$ error $(\approx 60 \mu \mathrm{H})$ for stretchable elements. Fig. 5(b) presents the computed value of $L_{11}$ for various external distances. One can see that the best selection for the number of layers is 14 and for external distances more than $60 \mathrm{~mm}$, the calculated value of inductance changes less than $5 \%(\approx 2.5 \mu \mathrm{H}$ increase). Selection of a reasonable value of 3-D elements will results in an acceptable computation time and accuracy. For example, in case of considering 14400 elements (72 sector in $\theta$ direction, ten ring in $Z$-direction, and 20 layer in radial direction) the total computation time was about $230 \mathrm{~s}$ using a PC system (CPU: 64 bit/3.1-GHz Intel i5-2400, RAM: $4.00 \mathrm{~GB}$ ). The resulted values of core dimension, number of winding turns, and thickness of insulator are used to implement the MWT prototype as is discussed in the next section. 


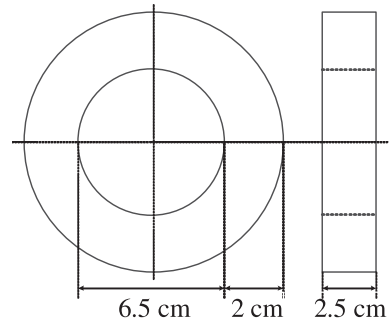

(a)

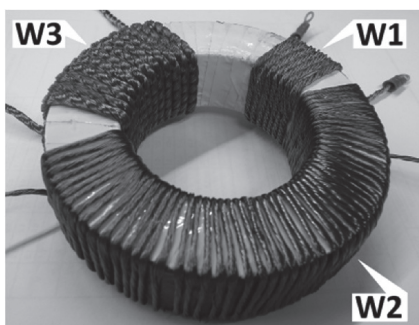

(b)
Fig. 6. (a) Dimensions of magnetic core. (b) Prototype of transformer and windings.

\section{Prototyping of the Transformer}

A prototype of three winding high-frequency toroidal transformer is implemented for a residential microgrid that we are developing. The first stage of the transformer design was selection of magnetic materials because core permeability factor was required for numerical design. The soft ferrite have been widely used in high-frequency converters due to their availability and low price although their saturation flux density is low $(0.3-0.5 \mathrm{~T})$ and results in the transformer large size. Amorphous alloy and nanocrystalline materials have high saturation flux density, high permeability, and low core loss. Comparing their characteristics show that nanocrystalline materials have lower core loss than the amorphous alloys although their saturation flux density $(0.8-1 \mathrm{~T})$ is much lower than that of amorphous alloys (1.4$1.6 \mathrm{~T})$ [11]. The Metglas amorphous alloy 2605SA1 strip of 25 -mm width and $20-\mu \mathrm{m}$ thickness made by Hitachi metals is finally selected taking into account specific core loss, maximum flux density, cost and availability. To develop the magnetic core, the Metglas 2605SA1 thin tape was glued with Araldite 2011 on the surface of each layer and wounded around a cylindrical frame with external diameter of $65 \mathrm{~mm}$. The developed core dimension and the transformer are presented in Fig. 6. The hysteresis loops and the core loss properties were measured experimentally. The excitation waveforms are generated by a TTiTGA1244 programmable waveform generator and are amplified using high-frequency signal amplifier AM3002. A power analyzer PM3000A is employed to measure the transformer power losses and Tektronix current measurement probe TCPA300 and high-voltage differential probe P5200 are used to observe the transformer waveforms as presented in Fig. 7(a). To measure the $B-H$ curve of developed core under $10-\mathrm{kHz}$ square-wave excitation current, winding one is selected as excitation coil and winding three as pickup coil. Considering almost uniform flux density inside the core, the magnetic field intensity $H$ can be calculated using Ampere's law as

$$
H=N_{A} i_{A}(t) / l_{e}
$$

where $N_{A}$ is the number of turns in excitation winding, $i(t)$ is the measured excitation current, and $l_{e}$ is the mean length of the toroidal core. To calculate the magnetic flux density in the core, Faraday's law should be applied using open-circuit voltage of

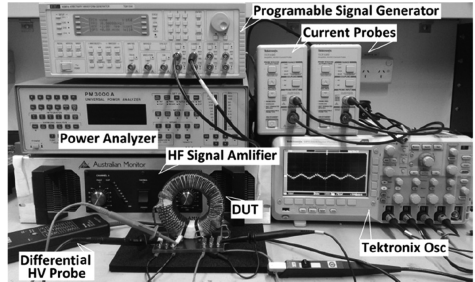

(a)

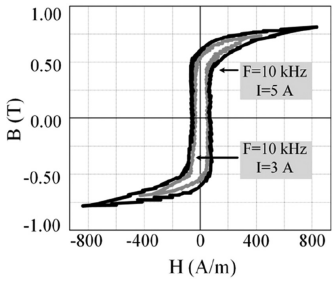

(b)

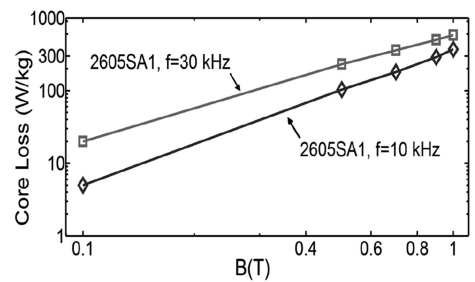

(c)
Fig. 7. (a) Experimental test platform. (b) Measured hysteresis loops the magnetic core for $f=10 \mathrm{kHz}, 3 \mathrm{~A}$, and $5 \mathrm{~A}$. (c) Measured core loss of Metglas 2605SA1 for $10-\mathrm{kHz}$ and $30-\mathrm{kHz}$ excitation currents.

the pickup coil

$$
B=\frac{1}{N_{B} A_{e}} \int V_{B}(t) d t
$$

where $N_{B}$ is the number of turns of pickup coil, $A_{e}$ is the cross-sectional area of magnetic core, and $V_{B}$ is the measured open-circuit voltage of the winding. The recorded measured data are used to plot the $B-H$ loop as shown in Fig. 7(b).

The core loss was measured for two cases of $10-\mathrm{kHz}$ and 30-kHz square-wave excitation currents as shown in Fig. 7(c). The total loss (core loss plus copper loss) was measured using a power analyzer. Considering almost equal ac and dc winding resistances in case of using Litz wires, the copper loss can be calculated easily and the core loss can be defined. The core loss was calculated using the Steinmetz equation as

$$
P_{\text {Coreloss }}=k f^{m} B^{n}
$$

where $f$ is frequency and $B$ is the magnitude of flux density. The resulted core loss characteristics are used to define the new coefficients $(k=6.21, m=1.60, n=1.64)$ for (11) under squarewave currents as the datasheet coefficients $(k=6.5, m=1.51$, $n=1.74$ ) are derived under sinusoidal test currents. To reduce the skin and proximity effects resulted in high-frequency currents, and consequently, reduce the ac resistance and winding loss, a Litz wire should be used for transformer windings. The minimum diameter of a single strand of a Litz wire, $d$ was defined using

$$
d=\sqrt{\frac{4 i}{J \pi S_{n}}}
$$

where $i$ is the winding current, $S_{n}$ is the number of isolated strands, and $J$ is the current density. In our design for all three windings, the Litz wires with diameter of $0.35 \mathrm{~mm}$ are considered. The number of twisted insulated strands for windings one, two, and three is $30(3 * 10), 18(3 * 6)$, and $45(3 * 3 * 5)$ and their number of turns resulted from design process is 7,53 , and 12 turns, respectively. The overall diameters of twisted wires for 
windings were measured approximately as $\mathrm{d}_{1} \approx 3 \mathrm{~mm}, \mathrm{~d}_{2} \approx 2$ $\mathrm{mm}$, and $d_{3} \approx 4 \mathrm{~mm}$. The experimental test desk of Fig. 7(a) was used to test the validity of design process and measure the transformer parameters. The test process is based on the method suggested in [32] due to the MWT high leakage inductance. The programmable signal generator, TTi-TGA1244, and the HF signal amplifier are used to generate the sinusoidal excitation currents. The standard short- and open-circuit tests have been used conventionally to determine the transformer inductive and resistive elements. It has been shown that short-circuit test has less accuracy when the winding resistances and the leakage reactances have significant values relative to the magnetizing impedance [32], [33]. It also will lose its accuracy in case of big difference between the primary and secondary leakage inductances and winding resistances due to winding geometries and configurations. On the other hand, it is necessary to have the same value of magnetic flux, and consequently, permeability factor in the core during all stages of experimental tests. It is difficult to maintain the same magnetizing current in the shortcircuit test because of parallel connection of the magnetizing branch and the secondary winding impedance during the test [32]. Therefore, the short-circuit test did not provide reliable results and was excluded from our test procedures. To measure the transformer leakage and self-inductances, the conventional open circuit and the differentially and cumulatively coupled tests are applied to the windings with one as the primary and three as the secondary. To discuss the experimental test process, windings one and three are selected as examples and the test procedure is applied similarly to other two pairs (windings one and two or windings two and three).

\section{A. Open-Circuit Test}

During this test, the test voltage set as $110 \mathrm{~V}$ at $10 \mathrm{kHz}$ was applied to winding one, while winding three was open circuited. The values of impedances are calculated using the normal equation of sinusoidal exited circuits [32]. The measured impedance including the real and imaginary parts is

$$
\begin{aligned}
Z_{1}(\mathrm{OC}) & =R_{1}(\mathrm{OC})+j \omega L_{1}(\mathrm{OC}) \\
& =\left(R_{W 1}+R_{m}\right)+j \omega\left(L_{l 1}+L_{13}\right) .
\end{aligned}
$$

At the second stage, the excitation current was applied to winding three with the other two windings open circuited [32]. The resultant impedance in this case is

$$
\begin{aligned}
Z_{3}(\mathrm{OC}) & =R_{3}(\mathrm{OC})+j \omega L_{3}(\mathrm{OC}) \\
& =\left(R_{W 3}+R_{m} n^{2}\right)+j \omega\left(L_{l 3}+n^{2} L_{13}\right)
\end{aligned}
$$

where $n$ is the turn ratio of winding three to winding one. The schematics of the test circuits are shown in Fig. 8(a) and (b).

\section{B. Series-Coupling Tests}

In this test, winding one is considered as the primary and winding three as the secondary. They are connected in series in differential or cumulative form as illustrated in Fig. 8(c) and (d), while winding two was open circuited. The series-coupling test is used to factor in the effects on the leakage and magnetizing

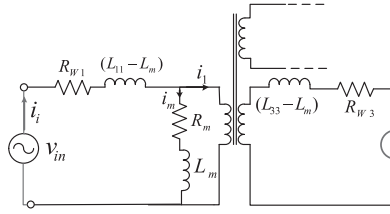

(a)

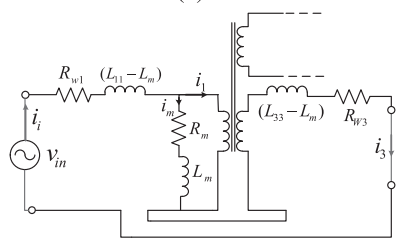

(c)

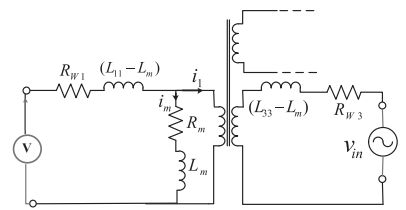

(b)

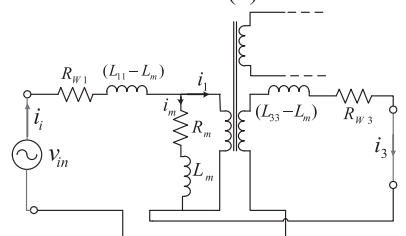

(d)
Fig. 8. Experimental tests of multiwinding transformer. (a) Secondary open-circuit test. (b) Primary open-circuit test. (c) Differentially coupled test. (d) Cumulatively coupled test.

inductances of core excitation [32]. Compared with the parallel coupling test, the measured inductances and resistances are the algebraic addition and subtraction of resistive and inductive elements of the transformer windings, which facilitates direct measurement by the meter. The input impedances measured in differential and cumulatively coupled tests are as ings, which facilitates direct measurement by the meter. The input impedances measured in differential and cumulatively coupled tests are

$$
\begin{aligned}
Z_{1}(\text { dif })= & R_{13}(\text { dif })+j \omega L_{13}(\text { dif }) \\
= & \left(R_{W 1}+R_{W 3}+(1-n)^{2} R_{m}\right) \\
& +j \omega\left(L_{l 1}+L_{l 3}+(1-n)^{2} L_{13}\right) \\
Z_{1}(\mathrm{Cum})= & R_{13}(\text { cum })+j \omega L_{13}(\text { cum }) \\
= & \left(R_{W 1}+R_{W 3}+(1+n)^{2} R_{m}\right) \\
& +j \omega\left(L_{l 1}+L_{l 3}+(1+n)^{2} L_{13}\right) .
\end{aligned}
$$

A combination of series-coupling and the open-circuit tests have been used to find the value of transformer parameters [32]. The resultant equations in terms of inductances $L_{1}(\mathrm{oc})$, $L_{3}$ (oc), $L_{13}$ (dif), and $L_{13}$ (cum) and the resistances $R_{1}$ (oc), $R_{3}$ (oc), $\quad R_{13}$ (dif), and $R_{13}$ (cum) and $R_{m}$ are tabulated in Table I. The resulted values of all three tabulated equations were quite consistent and their average is considered as the final value of the parameter. To guarantee the same value of magnetizing flux density into the core during all the tests, the following relationships should be considered for excitation current during various tests [32]. This will results in a consistent flux density in the core during all tests, and hence, an almost constant values for $L_{m}$ and $R_{m}$.

$$
I_{p(\mathrm{OC})}=n I_{s(\mathrm{OC})}=I_{\mathrm{dif}}(1-n)=I_{\mathrm{cum}}(1+n)
$$

where $I_{P}$ and $I_{\mathrm{s}}$ denote the primary and secondary currents, respectively. It should be noticed that during the series-coupling test the value of $L_{m}$ is equal to the mutual inductance of two windings under the test ( $L_{13}$ in this case). Fig. 9 shows the resultant values of inductances and resistances for windings one and three with the excitation current ranging from 0.5 to $4.5 \mathrm{~A}$. From Fig. 9(a), it is clear that as the winding current increases, the 
TABLE I

Equations of Model Parameters for Different Test Procedures

\begin{tabular}{|c|c|c|c|}
\hline Test Parameter & Differentially Coupled and open-Circuit Tests & Cumulatively Coupled and Open Circuit Tests & Combination of all Three Tests \\
\hline \multirow{2}{*}{$R_{W 1}$} & $(2 n-1) R_{1}(\mathrm{OC})-R_{3}(\mathrm{OC})+R_{13}(\mathrm{dif})$ & $(2 n+1) R_{1}(\mathrm{OC})+R_{3}(\mathrm{OC})-R_{13}(\mathrm{cum})$ & \multirow{2}{*}{$R_{1}(\mathrm{OC})-\frac{R_{13}(\mathrm{cum})-R_{13}(\mathrm{dif})}{4 n}$} \\
\hline & $2 n$ & $2 n$ & \\
\hline \multirow{2}{*}{$R_{W 3}$} & $(2-n) R_{3}(\mathrm{OC})-n R_{1}(\mathrm{OC})+n R_{13}($ dif $)$ & $(2+n) R_{3}(\mathrm{OC})+n R_{1}(\mathrm{OC})-n R_{13}(\mathrm{cum})$ & \multirow{2}{*}{$R_{3}(\mathrm{OC})-\frac{n R_{13}(\mathrm{cum})-n R_{13}(\mathrm{dif})}{4}$} \\
\hline & 2 & 2 & \\
\hline \multirow{2}{*}{$R_{m}$} & $R_{1}(\mathrm{OC})+R_{3}(\mathrm{OC})-R_{13}(\mathrm{dif})$ & $R_{13}(\mathrm{cum})-R_{1}(\mathrm{OC})-R_{3}(\mathrm{OC})$ & \multirow{2}{*}{$\frac{R_{13}(\mathrm{cum})-R_{13}(\text { dif })}{4 n}$} \\
\hline & $2 n$ & $2 n$ & \\
\hline \multirow{2}{*}{$L_{l 1}$} & $(2 n-1) L_{1}(\mathrm{OC})-L_{3}(\mathrm{OC})+L_{13}($ dif $)$ & $(2 n+1) L_{1}(\mathrm{OC})+L_{3}(\mathrm{OC})-L_{13}(\mathrm{cum})$ & \multirow{2}{*}{$L_{1}(\mathrm{OC})-\frac{L_{13}(\mathrm{cum})-L_{13}(\mathrm{dif})}{4 n}$} \\
\hline & $2 n$ & $2 n$ & \\
\hline \multirow{2}{*}{$L_{l 3}$} & $(2-n) L_{3}(\mathrm{OC})-n L_{1}(\mathrm{OC})+n L_{13}(\mathrm{dif})$ & $(2+n) L_{3}(\mathrm{OC})+n L_{1}(\mathrm{OC})-n L_{13}(\mathrm{cum})$ & \multirow{2}{*}{$L_{3}(\mathrm{OC})-\frac{n L_{13}(\mathrm{cum})-n L_{13}(\mathrm{dif})}{4}$} \\
\hline & 2 & 2 & \\
\hline \multirow{2}{*}{$L_{13}$} & $\underline{L_{1}(\mathrm{OC})-L_{3}(\mathrm{OC})-L_{13}(\mathrm{dif})}$ & $\underline{L_{13}(\mathrm{cum})-L_{1}(\mathrm{OC})-L_{3}(\mathrm{OC})}$ & \multirow{2}{*}{$\frac{L_{13}(\mathrm{cum})-L_{13}(\text { dif })}{4 n}$} \\
\hline & $2 n$ & $2 n$ & \\
\hline
\end{tabular}

mutual inductance between windings one and three decreases, while their leakage inductance increases. On the other hand, Fig. 9(b) shows that the value of equivalent core loss resistance will increase with any increase in the test current while the winding resistances are not dependent on the test current and remain constant. To find the accuracy of implementation process, the experimentally measured inductances are compared with the numerically calculated ones. Fig. 9(c) shows the measured values of leakage and self-inductance of winding one with excitation current of $5 \mathrm{~A}$. Comparing measured values with numerically calculated inductances shows that the resulted error is reduced to less than $15 \%$ considering 144003 -D elements for magnetic structure. A further reduction of error to $10 \%$ was achieved by increasing the number of elements to 19440 elements although it increased the computation time considerably (about $11 \mathrm{~min}$ ). The resultant error can be attributed to various experimental factors that cannot be included properly in the numerical analysis, such as stacking factor and windings distribution.

\section{VAlidity Test of Designed TRANSFormeR}

The major parasitic effects are due to the skin effect, proximate effect, and stray capacitance. In this research, the skin and proximity effects are considered in the design process and the effect of stray capacitance is safely ignored because of their small value and relatively low switching frequency, about $10 \mathrm{kHz}$. The numerical model of MWT based on the measured parameters was simulated under different load conditions for medium-frequency range using MATLAB/Simulink. The results are compared with the experimental tests to validate the modeling process. As the windings current in our application are nonsinusoidal including high-frequency harmonics, the se- lected frequency range covers the fundamental frequency of current $(10 \mathrm{kHz})$ and its harmonics $(30,50$, and $70 \mathrm{kHz})$.

The excitation current was applied to winding one of the MWT as the primary and winding three as the secondary where the output signals for entire frequency range were recorded. The experimental tests were carried out for three cases of inductive load, capacitive load, and short-circuit conditions to factor in both mutual and leakage inductive effects. A sinusoidal voltage of $150 \mathrm{~V}(\mathrm{rms})$ with variable frequency changes from 10 to $150 \mathrm{kHz}$ generated by programmable the signal generator and amplified by the HF signal amplifier was used as excitation source. The test process was carried out for other pairs of windings and the results were consistent. Fig. 10(a)-(c) shows the equivalent circuit of windings one and three under the test for inductive load, capacitive load, and short-circuit condition, while winding two is open circuited. The transfer function of circuit in case of capacitive load can be defined as

$$
\begin{aligned}
& \frac{V_{O}(S)}{V_{\text {in }}(S)}= \\
& \frac{Z}{\left[R_{W 1}+\left(L_{11}-L_{m}\right) S+Z\right]\left[1+S C^{\prime} R_{W 3}^{\prime}+S^{2} C^{\prime}\left(L_{33}^{\prime}-L_{m}\right)\right]}
\end{aligned}
$$

where $\mathrm{Z}$ can be found as

$$
[Z]^{-1}=\left[R_{m}+S L_{m}\right]^{-1}+\left[R_{W 3}^{\prime}+S\left(L_{33}^{\prime}-L_{m}\right)+1 / S C^{\prime}\right]^{-1}
$$

In case of inductive load, the transfer function of secondary to primary voltage and impedance $Z$ can be calculated as (20) and (21), shown at the bottom of the page.

$$
\begin{aligned}
& \frac{V_{O}(S)}{V_{\mathrm{in}}(S)}=\frac{Z^{\prime}\left(r_{O}^{\prime}+L_{O}^{\prime} S\right)}{\left[R_{W 1}+\left(L_{11}-L_{m}\right) S+Z^{\prime}\right]\left[r_{O}^{\prime}+R_{W 3}^{\prime}+S\left(L_{O}^{\prime}+L_{33}^{\prime}-L_{m}\right)\right]} \\
& {\left[Z^{\prime}\right]^{-1}=\left[R_{m}+S L_{m}\right]^{-1}+\left[r_{o}^{\prime}+R_{W 3}^{\prime}+S\left(L_{O}^{\prime}+L_{33}^{\prime}-L_{m}\right)\right]^{-1}}
\end{aligned}
$$




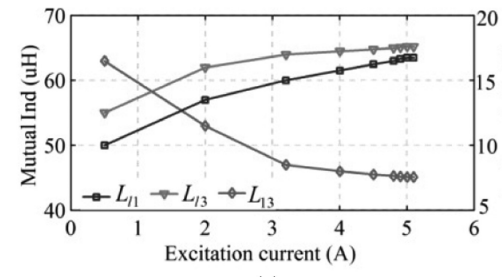

(a)

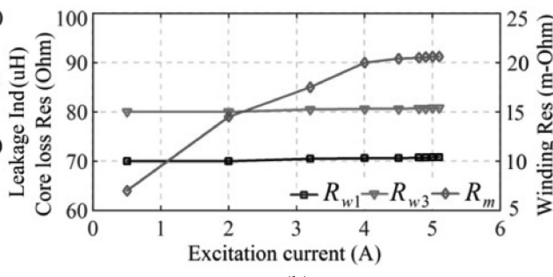

(b)

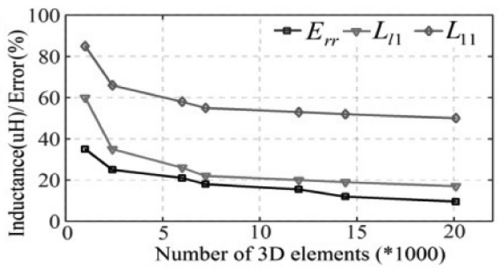

(c)

Fig. 9. Measured values of (a) inductances, (b) resistances for windings one and three, and (c) leakage inductance and self-inductance of winding one and percentage of error versus number of 3-D elements.

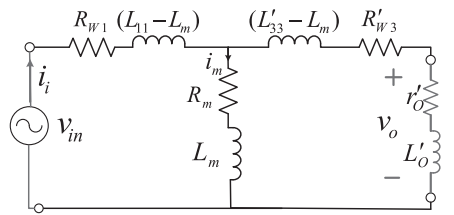

(a)

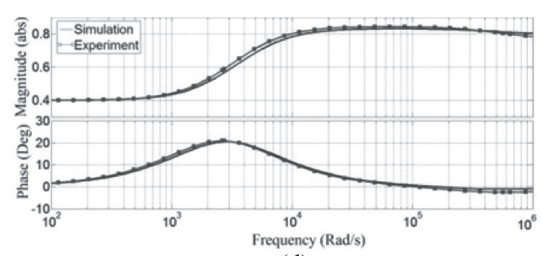

(d)

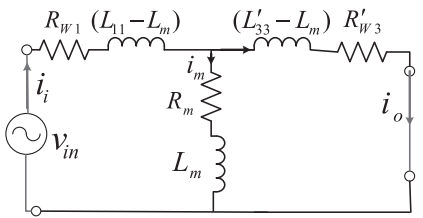

(b)

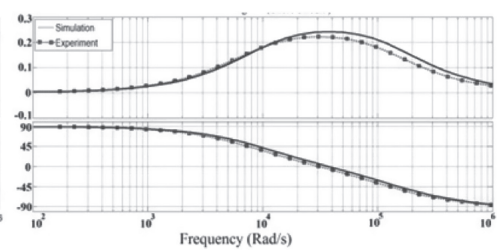

(e)

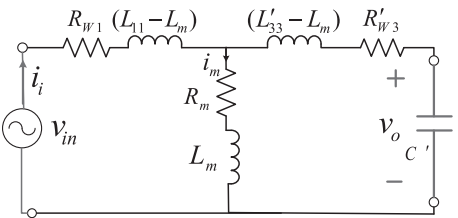

(c)

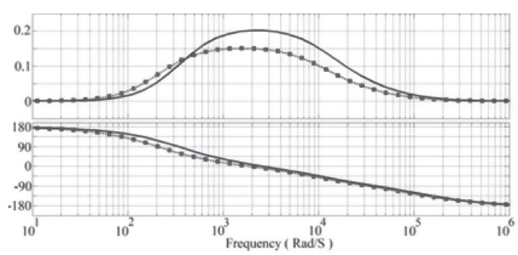

(f)

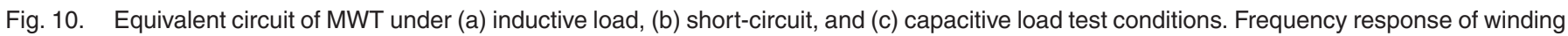
one to three of MWT for cases (d) inductive load, (e) short-circuit, and (f) capacitive load conditions (solid line is simulation and dashed line is experimental results).

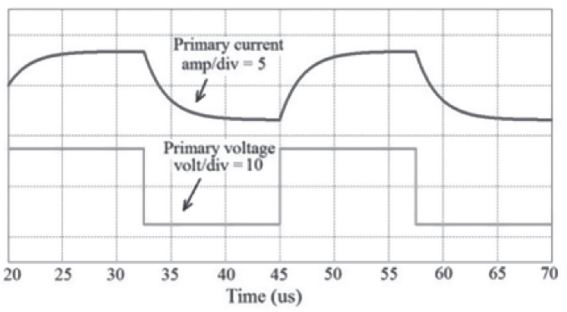

(a)

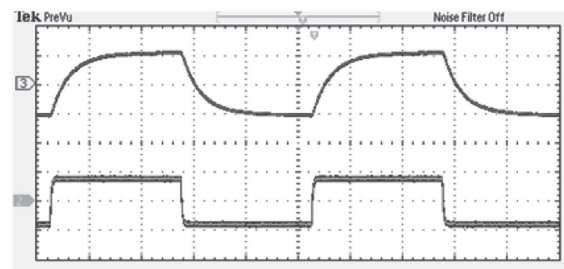

(d)

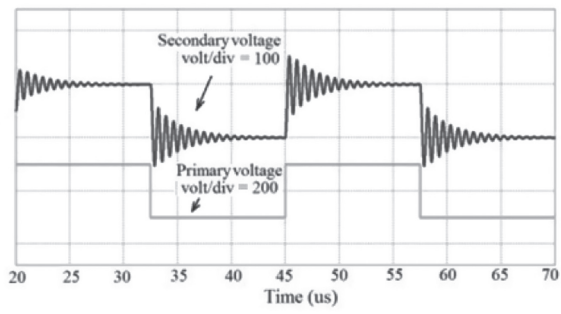

(b)

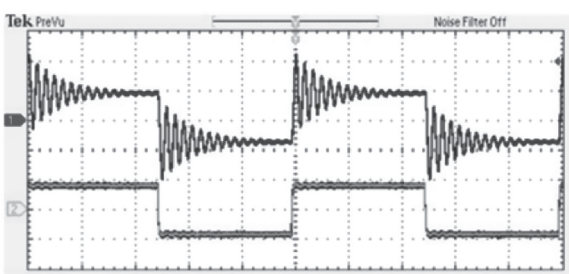

(e)

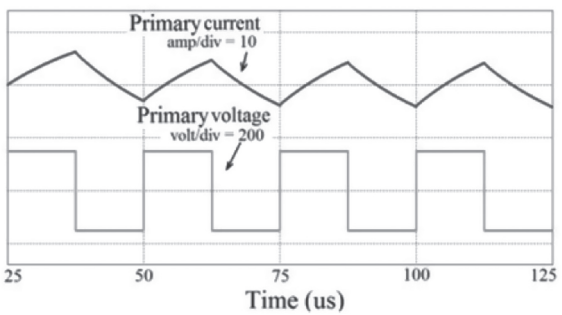

(c)

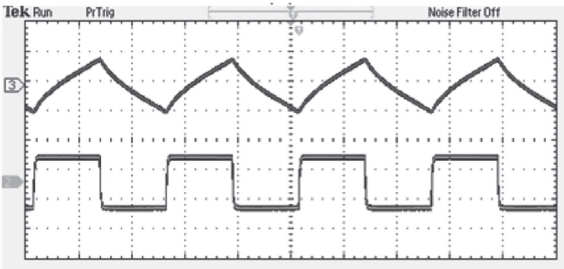

(f)

Fig. 11. Simulation results of MWT model for square-wave excitation using PSIM for cases of (a) short-circuit condition, (b) capacitive load, and (c) inductive load and experimental test results for (d) short-circuit condition, (e) capacitive load, and (f) inductive load conditions, $\mathrm{CH}$ 1: Secondary voltage, $\mathrm{CH}$ 2: Primary voltage, $\mathrm{CH} 3$ : Primary current.

The last test was carried out under the short-circuit condition and the transfer function and impedance $\mathrm{Z}$ can be defined as

$$
\begin{aligned}
\frac{I_{O}(S)}{V_{\text {in }}(S)} & =\frac{Z}{\left[R_{W 1}+\left(L_{11}-L_{m}\right) S+Z\right]\left[R_{W 3}^{\prime}+S\left(L_{33}^{\prime}-L_{m}\right)\right]} \\
{[Z]^{-1} } & =\left[R_{m}+S L_{m}\right]^{-1}+\left[R_{W 3}^{\prime}+S\left(L_{33}^{\prime}-L_{m}\right)\right]^{-1} .
\end{aligned}
$$

Comparing experimental and simulation results shows that the simulated model adapts very well with experimental test results for all three types of loads. Looking at Fig. 10(d)-(f) shows that the difference between the simulated and experimental results is small for medium-frequency range as the modeling process and experimental tests are carried out for this range. The measured steady-state core and the winding temperatures were in the range of $40-60{ }^{\circ} \mathrm{C}$ during the frequency response tests 
under the nominal load conditions. Within this temperature range the effect of temperature on winding resistance is negligible. On the other hand the magnetic characteristics of amorphous core material, used for the transformer are almost independent to temperature according to the data provided by manufacturer, therefore, the effect of temperature on the transformer parameters were negligible. Fig. 11 compares the experimental and simulation results in the time domain with a $40-\mathrm{kHz}$ square-wave excitation. It can be seen that the simulations and experimental wave forms show a very good agreement for all three types of load conditions. Based on the results the suggested method for design and characterization of MWTs is validate for mediumfrequency range where parasitic effect of stray capacitors are negligible and the operation point of magnetic component is in linear area of $B-H$ curve.

\section{CONCLUSION}

Due to the application of MWTs as a common magnetic link in integration of renewable energy sources, they have attracted considerable research interest. This paper covered three stages of design, prototyping, and experimental test of a high-frequency toroidal MWT. The RNM was used to design the transformer based on the required specifications due to the low computation time. A prototype transformer was developed using amorphous magnetic materials and the transformer parameters including leakage and mutual inductances and resistances are measured applying open-circuit and series-coupling tests. A comparison between the simulation and experimental test results under different loads within the medium-frequency range validated both design and modeling procedures.

\section{REFERENCES}

[1] D. Gunasekaran and L. Umanand, "Integrated magnetics based multi-port bidirectional DC-DC converter topology for discontinuous-mode operation," IET Power. Electron., vol. 5, no. 7, pp. 935-944, Aug. 2012.

[2] M. R. Islam, Y. G. Guo, Z. W. Lin, and J. G. Zhu, "An amorphous alloy core medium frequency magnetic-link for medium voltage PV inverters," J. Appl. Phys., vol. 115, no. 17, pp. 17E710-17E710-3, May 2014.

[3] M. Ishigaki, K. Ito, S. Tomura, and T. Umeno, "A new isolated multi-port converter using interleaving and magnetic coupling inductor technologies," in Proc. 28th Annu. IEEE Appl. Power Electron Conf. Expo., Long Beach, CA, USA, 2013, pp. 1068-1074.

[4] Y. M. Chen, Y. C. Liu, and F. Y. Wu, "Multi-input DC/DC converter based on the multiwinding transformer for renewable energy applications," IEEE Trans. Ind. Appl., vol. 38, no. 4, pp. 1096-1104, Jul./Aug. 2002.

[5] M. R. Islam, G. Lei, Y. Guo, and J.Zhu, "Optimal design of high-frequency magnetic links for power converters used in grid-connected renewable energy systems," IEEE Trans. Magn., vol. 50, no. 11, pp. 1-4, Nov. 2014.

[6] I. O. Lee, "Hybrid DC-DC converter with phase-shift or frequency modulation for NEV battery charger," IEEE Trans. Ind. Electron., vol. 63, no. 2, pp. 884-893, Feb. 2016.

[7] J. Zhang, H. Wu, X. Qin, and Y. Xing, "PWM plus secondary-side phase-shift controlled soft-switching full-bridge three-port converter for renewable power systems," IEEE Trans. Ind. Electron., vol. 62, no. 11, pp. 7061-7072, Nov. 2015.

[8] Y. Shi, R. Li, Y. Xue, and H. Li, "Optimized operation of current-fed dual active bridge DC-DC converter for PV applications," IEEE Trans. Ind. Electron., vol. 62, no. 11, pp. 6986-6995, Nov. 2015.

[9] H. Tao, J. L. Duarte, and M. A. M. Hendrix, "Three-port triple-half-bridge bidirectional converter with zero-voltage switching," IEEE Trans. Power Electron., vol. 23, no. 2, pp. 782-792, Mar. 2008.

[10] H. Tao, A. Kotsopoulos, J. L. Duarte, and M. A. M. Hendrix, "Family of multiport bidirectional DC-DC converters," Proc. Inst. Elect. Eng., vol. 153 , no. 3, pp. 451-458, 2006.
[11] M. R. Islam, Y. G. Guo, and J. G. Zhu, "High-frequency link multilevel cascaded medium-voltage converter for direct grid integration of renewable energy systems," IEEE Trans. Power Electron., vol. 29, no. 8, pp. 4167-4182, Aug. 2014.

[12] C. Zhao and J. W. Kolar, "A novel three-phase three-port UPS employing a single high-frequency isolation transformer," in Proc. 35th IEEE Power Electron. Spec. Conf., vol. 6, 2004, pp. 4135-4141.

[13] F. de León, S. Purushothaman, and L. Qaseer, "Leakage inductance design of toroidal transformers by sector winding," IEEE Trans. Power Electron., vol. 29, no. 1, pp. 473-480, Jan. 2014.

[14] I. Hernandez, F. de Leon, and P. Gomez, "Design formulas for the leakage inductance of toroidal distribution transformers," IEEE Trans. Power Del., vol. 26, no. 4, pp. 2197-2204, Oct. 2011.

[15] A. J. Binnie and T. R. Foord, "Leakage inductance and interwinding capacitance in toroidal ratio transformers," IEEE Trans. Instrum. Meas., vol. 16, no. 4, pp. 307-314, Dec. 1967.

[16] W. G. Hurley and D. J. Wilcox, "Calculation of leakage inductance in transformer windings," IEEE Trans. Power Electron., vol. 9, no. 1, pp. 121-126, Jan. 1994.

[17] A. Dauhajre and R. D. Middlebrook, "Modelling and estimation of leakage phenomena in magnetic circuits," in Proc. 17th Annu. IEEE Power Electron. Spec. Conf., Vancouver, Canada, 1986, pp. 213-226.

[18] J. Wang, A. F. Witulski, J. L. Vollin, T. K. Phelps, and G. I. Cardwell, "Derivation, calculation and measurement of parameters for a multiwinding transformer electrical model," in Proc. 14th Appl. Power Electron. Conf. Expo., Dallas, TX, USA, 1999, vol. 1, pp. 220-226.

[19] G. Skutt, F. C. Lee, R. Ridley, and D. Nicol, "Leakage inductance and termination effects in a high-power planar magnetic structure," in Proc. 9th Annu. Appl. Power Elec. Conf. Expo., vol. 1, Orlando, FL, USA, 1994, pp. 295-301.

[20] H. Tao, A. Kotsopoulos, J. L. Duarte, and M. A. M. Hendrix, "Multiinput bidirectional DC-DC converter combining DC-link and magneticcoupling for fuel cell systems," in Proc. Ind. Appl. Conf., 2005, vol. 3, pp. 2021-2028.

[21] C.Zhao, S. D. Round, and J. W. Kolar, "An isolated three-port bidirectional DC-DC converter with decoupled power flow management," IEEE Trans. Power Electron., vol. 23, no. 5, pp. 2443-2453, Sep. 2008.

[22] H. Tao, A. Kotsopoulos, J. L. Duarte, and M. A. M. Hendrix, "A softswitched three-port bidirectional converter for fuel cell and supercapacitor applications," in Proc. 36th IEEE Power Electron. Spec. Conf., Recife, Brazil, 2005, pp. 2487-2493.

[23] H. Tao, A. Kotsopoulos, J. L. Duarte, and M. A. M. Hendrix, "Triplehalf-bridge bidirectional converter controlled by phase shift and PWM,' in Proc. 21st Annu. IEEE Appl. Power Electron. Conf. Expo., Dallas, TX, USA, 2006, pp. 1256-1262.

[24] J. Worotynski, M. Turowski, and E. Worotynska, "Generation of an optimal reluctance network model for fast 2-D and 3-D simulations of electromagnetic devices," in Proc. 2nd Int. Conf. Comput. Electromagn., 1994, London, U.K., pp. 116-119.

[25] F. Janet, J. L. Coulomb, C. Chillet, and P. Mas, "Magnetic moment and reluctance network mixed method applied to transformer modeling," IEEE Trans. Magn., vol. 41, no. 5, pp. 1428-1431, May 2005.

[26] D. Vanoost, H. De Gersem, J. Peuteman, G. Gielen, and D. Pissoort, "Twodimensional magnetostatic finite-element simulation for devices with a radial symmetry," IEEE Trans. Magn., vol. 50, no. 5, pp. 1-4, May 2014.

[27] B. Auchmann, B. Flemisch, and S. Kurz, "A discrete 2-D formulation for 3-D field problems with continuous symmetry," IEEE Trans. Magn., vol. 46, no. 8, pp. 3508-3511, Aug. 2010.

[28] Z. Zhanxin, X. Dexin, W. Gang, Z. Yanli, and Y. Xiuke, "Computation of 3-D magnetic leakage field and stray losses in large power transformer," IEEE Trans. Magn., vol. 48, no. 2, pp. 739-742, Feb. 2012.

[29] S. Ghalavand, V. Zadeh, and Isfahani, "An improved magnetic equivalent circuit model for iron-core linear permanent-magnet synchronous motors," IEEE Trans. Magn., vol. 46, no. 1, pp. 112-120, Jan. 2010.

[30] M. Amrhein and P. T. Krein, "3-D magnetic equivalent circuit framework for modeling electromechanical devices," IEEE Trans. Energy Convers., vol. 24, no. 2, pp. 397-405, Jun. 2009.

[31] S. V. Kulkarni and S. A. Khaparde, Transformer Engineering Design \& Practice. New York, NY, USA: Marcel Dekker, 2004.

[32] J. G. Hayes, D. Cashman, M. G. Egan, and T. O’Donnell, "Comparison of test methods for characterization of high-leakage two-winding transformers," IEEE Trans. Ind. Appl. vol. 45, no. 5, pp. 1729—1741, Sep./Oct. 2009.

[33] J. G. Hayes, N. O’Donovan, M. G. Egan, and T. O’Donnell, "Inductance characterization of high-leakage transformers," in Proc. IEEE Appl. Power Electron. Conf., 2003, pp. 1150-1156. 


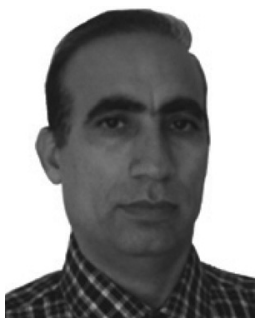

Mohammad Jafari (M'12) received the B.Sc. degree from Shiraz University, Shiraz, Iran, in 1998, and the M.Sc. degree from Guilan University, Rasht, Iran, in 2001, both in electrical engineering. He is currently working toward the Ph.D. degree at the University of Technology Sydney (UTS), Sydney, Australia.

From 2001 to 2011 , he contributed to the design and development of industrial power electronic projects. He has contributed to the design and development of many industrial power electronic projects. Since 2012, he has been with the UTS, as a Lecturer His current research interests include power electronic converters, renewable energy systems, and smart grids.

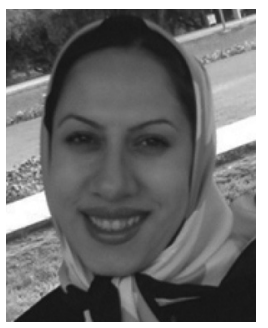

Zahra Malekjamshidi (S'13) received the B.Sc. and M.Sc. degrees from Shiraz University, Shiraz, Iran, in 1998, and 2001, respectively, both in electrical engineering. She is currently working toward the Ph.D. degree at the University of Technology Sydney, Sydney, Australia.

Her research interests include matrix converters, dc-dc converters, renewable energy technologies, and smart grids.

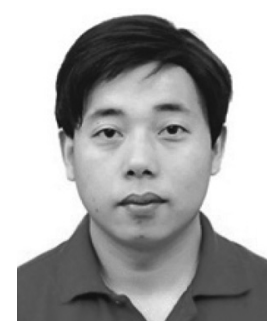

Gang Lei (M'14) received the B.S. degree in mathematics from Huanggang Normal University, Huanggang, China, in 2003, and the M.S. degree in mathematics and the Ph.D. degree in electrical engineering from Huazhong University of Science and Technology, Wuhan, China, in 2006 and 2009, respectively.

$\mathrm{He}$ is currently a Lecturer at the University of Technology Sydney, Sydney, Australia. His current research interests include electromagnetic inverse problems, design optimization of electri-

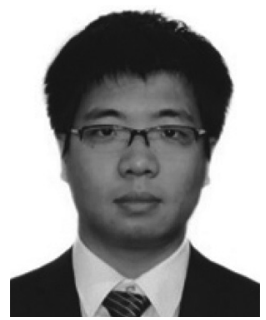

Tianshi Wang received the B.E. degree from Harbin Institute of Technology, Harbin, China, in 2008 , and the M.E. degree from the University of Technology Sydney, Sydney, Australia, in 2013, where he is currently working toward the Ph.D. degree.

His research interests include design, optimization, and advanced control algorithms of ac motor drives, optimal pulse width modulation, and advanced digital control with real-time implementation.

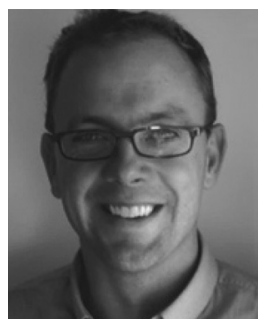

Glenn Platt received the Ph.D. degree from the University of Newcastle, Newcastle, Australia.

He leads the Grids and Energy Efficiency program within CSIRO's Energy Flagship, Newcastle, focusing on solving the energy challenges of consumers, business, and grids. The program's work ranges from energy storage, electric vehicles, smart grids, and the integration of largescale solar systems, through to solar cooling, energy management systems, economics and understanding people's response, and uptake of particular low-carbon energy options.

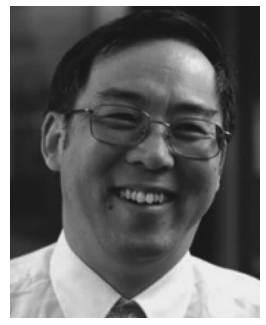

Jianguo Zhu (S'93-M'96-SM'03) received the B.E. degree from Jiangsu Institute of Technology, Jiangsu, China, in 1982, the M.E. degree from Shanghai University of Technology, Shanghai, China, in 1987, and the Ph.D. degree from the University of Technology Sydney (UTS), Sydney, Australia, in 1995.

$\mathrm{He}$ is currently a Professor of electrical engineering and the Head of the School of Electrical, Mechanical and Mechatronic Systems, UTS. His research interests include electromagnetics, magnetic properties of materials, electrical machines and drives, power electronics, and renewable energy systems. 